\title{
Comparing methods to estimate the proportion of turbine-induced bird and bat mortality in the search area under a road and pad search protocol
}

\author{
Joseph Duggan Maurer $^{1}$ D $\cdot$ Manuela Huso $^{2,3} \cdot$ Daniel Dalthorp $^{2}$. \\ Lisa Madsen ${ }^{3}$. Claudio Fuentes ${ }^{3}$
}

Received: 3 December 2018 / Revised: 2 September 2020 / Accepted: 19 September 2020 / Published online: 30 November 2020

(c) The Author(s) 2020

\begin{abstract}
Estimating bird and bat mortality at wind facilities typically involves searching for carcasses on the ground near turbines. Some fraction of carcasses inevitably lie outside the search plots, and accurate mortality estimation requires accounting for those carcasses using models to extrapolate from searched to unsearched areas. Such models should account for variation in carcass density with distance, and ideally also for variation with direction (anisotropy). We compare five methods of accounting for carcasses that land outside the searched area (ratio, weighted distribution, non-parametric, and two generalized linear models $(\mathrm{glm})$ ) by simulating spatial arrival patterns and the detection process to mimic observations which result from surveying only, or primarily, roads and pads (R\&P) and applying the five methods. Simulations vary R\&P configurations, spatial carcass distributions (isotropic and anisotropic), and per turbine fatality rates. Our results suggest that the ratio method is less accurate with higher variation relative to the other four methods which all perform similarly under isotropy. All methods were biased under anisotropy; however, including direction covariates in the $g l m$ method substantially reduced bias. In addition to comparing methods of accounting for unsearched areas, we suggest a semiparametric bootstrap to produce confidence-based bounds for the proportion of carcasses that land in the searched area.
\end{abstract}

\section{Handling Editor: Bryan F. J. Manly}

$凶 \quad$ Joseph Duggan Maurer

JosephMaurer7@gmail.com,jmaurer@wlgore.com

$\bowtie \quad$ Manuela Huso

mhuso@usgs.gov

1 W. L. Gore \& Associates, 4250 W Kiltie Ln, Flagstaff, AZ 86005, USA

2 US Geological Survey, Forest and Rangeland Ecosystem Science Center, 777 NW 9th Street, Suite 400, Corvallis 97330, OR, USA

3 Department of Statistics, Oregon State University, 239 Weniger Hall, Corvallis 97331, OR, USA 
Keywords Density-weighted proportion - Detection bias · R\&P · Wildlife mortality · Anisotropy

\section{Introduction}

Impacts to birds (particularly raptors) from collisions with wind turbines have been of concern in the U.S. since the early 1990s (Orloff and Flannery 1992) and impacts to bats since the early-2000s (Kerns and Kerlinger 2004). The magnitude of the impact is typically assessed through post-construction monitoring (PCM) (Howell and DiDonato 1991; Kunz et al. 2007; Arnett et al. 2008; Strickland et al. 2011). PCM to estimate mortality - the total number of turbine-induced bird or bat fatalities occurring over a specified period of time at individual wind projects-typically involves conducting carcass surveys, where human observers (or well-trained human-dog teams) search for carcasses within plots centered at randomly selected turbines (US Fish and Wildlife Service 2012). Under ideal circumstances, all carcasses from the sampled turbines land and remain in the designated search plots, and the entire area within each plot is searchable. In addition, under ideal conditions, all carcasses are detected by searchers with probability $=1$.

In practice, search conditions tend to be far from this ideal and counts of observed turbine-caused wildlife fatalities will necessarily underestimate total mortality (Huso 2011), because not all turbine-caused fatalities are observed in the search process and each carcass may have a unique probability of detection depending on when or where it falls and its specific characteristics (e.g., size or color). Carcasses can be removed by scavengers very soon after they arrive (Bernardino et al. 2011; Bispo et al. 2013; Flint et al. 2010), and humans and even the best scent-dogs fail to detect some carcasses (Homan et al. 2001; Arnett 2006; Mathews et al. 2013; Beebe et al. 2016; Reyes et al. 2016). Trials designed to estimate the probability that a carcass persists to the next search must be flexible enough to allow for non-constant removal and must account for potential differences due to carcass size, season, vegetation density in which the carcass lands, and other carcass-specific factors (Bernardino et al. 2011; Bispo et al. 2013; Turner et al. 2017). Likewise, searcher efficiency-the ability of a searcher to find a carcass that is present-may change in time (Wolpert 2013). The number of trial carcasses necessary to adequately estimate the effects of each of these factor combinations can be expensive. For example, placing 50 trial carcasses, as recommended by Strickland et al. (2011), in each of 4 size classes, 4 visibility/vegetation classes, and 4 seasons would result in 3200 total carcasses during a year and could potentially attract scavengers to the site.

The maximum distance at which a carcass can land from the turbine is unknown (Hull and Muir 2010), but empirical evidence suggests that species as small as bats can land $\geq 90 \mathrm{~m}$ from the turbine (Erickson et al. 2003; Chatfield et al. 2009) (Fig. 1). Extending the most commonly implemented plot shapes, square or circular (US Fish and Wildlife Service 2012), to or past $90 \mathrm{~m}$ is expensive and inefficient, because area increases with the square of the distance searched, but even with constant numbers within each distance class, carcass density (carcasses per unit area) decreases (Fig. 1). 

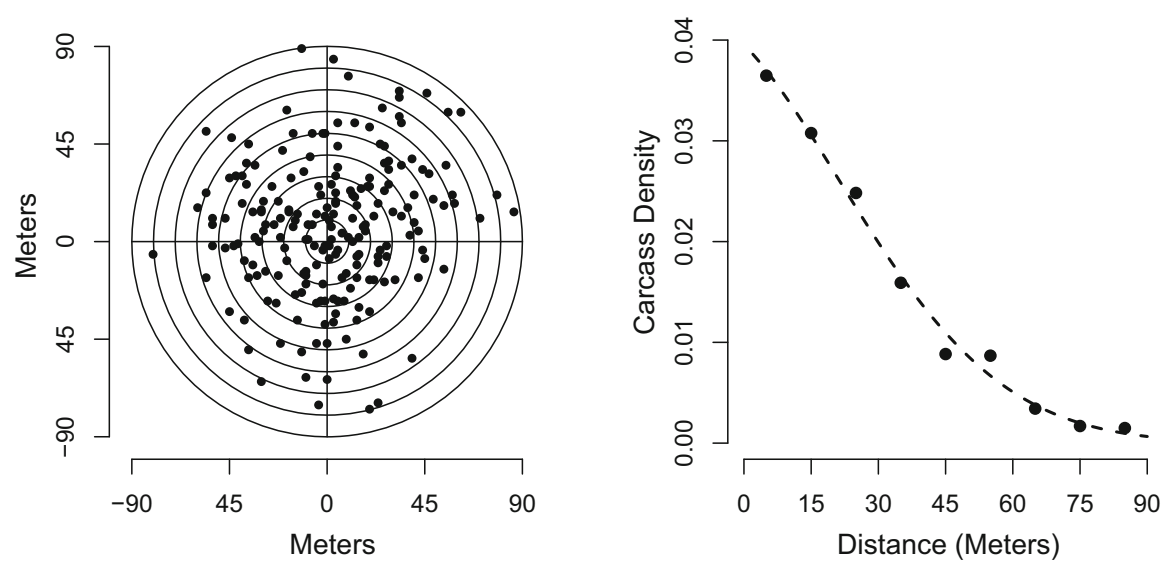

Fig. 1 (Left) Location with respect the nearest turbine of 200 carcasses observed on plots cleared of vegetation out to $90 \mathrm{~m}$ from April to November of 2013 at a wind project in the midwestern US. (Right) Observed (dots) carcass density (number of carcasses per meter ${ }^{2}$ ) for each 10-m annulus and estimated carcass density (dashed line). Poisson regression was used to obtain the fitted curve assuming the log mean carcass density follows a quadratic function of distance

Furthermore, even if the designated search plot encompasses the entire population of carcasses, thick vegetation, crops, dangerous terrain, and rivers or lakes within the plot boundaries can make surveying the entire plot impractical. Accurate estimation of mortality requires accounting for unsearched areas by estimating the proportion of total carcasses that landed within the searched area (Jain 2005; Huso and Dalthorp 2014; Huso et al. 2017), not the proportion of the designated plot area that was searched. A method to accurately estimate the probability density of carcasses at any distance and direction from the turbine would enable accurate estimation of the proportion of carcasses falling inside the searched area of plots of varying shapes and sizes, accommodating the site-specific conditions that might prevent full searches of standard plots.

Estimators of mortality, $M$, the total number of turbine-caused fatalities at a wind project over a specified period of time, have primarily been Horvitz-Thompson type estimators where observed carcass counts are divided by an estimated probability of detecting a carcass (Shoenfeld 2004; Huso 2011; Huso et al. 2012; Korner-Nievergelt et al. 2013; Wolpert 2015; Dalthorp et al. 2018), but see Etterson (2013) and Dalthorp et al. (2017) for alternative approaches. Although detection probability must account for all the major sources of imperfect detection outlined above, in this paper we focus on estimating one aspect: the proportion of carcasses landing within the searched area, $a$. Because the magnitude and variance of $a$ can greatly influence the precision with which $M$ is estimated, in this study we look at accuracy and precision of estimating both $a$ and $M$. To isolate the influence of the magnitude and uncertainty of $\hat{a}$ we evaluate its properties under idealized conditions where other detection factors are assumed known, but realistic in their magnitudes.

We consider two primary factors that could influence estimates of $a$ : (i) the mortality rate at each turbine, which in turn influences the number of carcasses discovered with 
which to fit models of $a$; and (ii) the spatial distribution of carcasses around each turbine relative to the searched area that in turn can influence the number of carcasses discovered with which to fit models of $a$, particularly if prevailing winds or other factors cause anisotropic spatial distributions.

In practice, resources available to conduct PCM are limited, and trade-offs must be made between the size of the search plot beneath each turbine and the fraction of turbines that can be visited (Dalthorp et al. 2017): the larger the search plot, the fewer turbines that can be sampled for a fixed monitoring budget. In addition, larger search plots will often comprise several visibility/vegetation classes with different probabilities of carcass persistence or searcher efficiency within each, incurring additional expense to conduct trials to estimate these probabilities within each class. In this context, it would be logical to consider limiting searching to areas in a single visibility/vegetation class (where carcass density or probability of detection is highest and consequently, variance of estimated mortality within the searched area is likely lowest) and rely on models of the proportion of carcasses landing within the searched area to extrapolate to the unsearched areas at the site. Such an approach would enable researchers to design carcass surveys that deviate from traditional square or circular plots and are conducive to increasing the maximum distance searched from the turbine without sacrificing efficiency. Road and pad (R\&P) surveys represent a convenient form of implementation of this methodology.

In principle, an R\&P search protocol has many potential advantages. The critical question is how reliably total mortality $M$ can be estimated from data primarily (or entirely) collected on roads and pads. The general approach is to first estimate the number of carcasses arriving on R\&P and then divide by the estimated proportion of carcasses falling on R\&P, $\hat{a}$. Ratio estimation (Thompson 2012) has also been utilized to obtain $\hat{a}$ (Good et al. 2011) by clearing vegetation from a small number of square or circular plots out to a specified distance, exhaustively searching the entire cleared areas, and calculating the proportion of the total carcasses found on R\&P. A potentially more robust and less costly alternative might be to model the distribution of carcasses as a function of distance (and possibly direction) from turbines and integrate over the R\&P. Such methods have been implemented by Arnett et al. (2009), Huso and Dalthorp (2014), and Hallingstad et al. (2018).

In this paper we compare the performance of five different estimators of $a$ under a range of conditions that might be found in practice. For example, the configurations of roads might be along the cardinal directions in flat agricultural areas, but along ridgelines in more rugged terrain. The reported average number of carcasses per turbine can vary considerably among facilities, which affects the number of carcasses observed on R\&P. Spatial carcass distributions can vary in unpredictable ways or may be highly dependent on direction of predominant winds. Comparisons focus on both potential bias and precision of each method for estimating $a$, and $M$ from it, under varying conditions. In Sect. 2, we describe five methods for estimating the proportion of carcasses that fell within the searched areas: four methods developed from concepts proposed by Jain (2005), Good et al. (2011), Arnett et al. (2009) and Huso and Dalthorp (2014), and one novel method using a parametric weighted distribution. In Sect. 3 we describe our simulation of spatial distributions of carcasses and the search process under a range of conditions. In Sect. 4 we evaluate the statistical properties of these 
methods. In Sect. 5 we propose a semi-parametric bootstrap method to capture the uncertainty associated with estimating $a$, the proportion of carcasses falling within searched area boundaries. We conclude with a general discussion of the performance of the methodologies and potential limitations of estimation from R\&P surveys in Sect. 6.

\section{Methods of accounting for unsearched area}

After colliding with a moving turbine blade, birds and bats fall to the ground in variable patterns that depend on several factors, including location of the strike along the blade, speed of the blade, wind speed, body weight, wing span, and possibly even animal movement after getting struck (for example, injured animals flapping in the air or crawling to relative safety after hitting the ground). The result is a spatial distribution of carcass density that is not constant but generally decreases with increasing distance from the turbine (Fig. 1). Because density is not constant, models that account for non-constant density must be employed to estimate the proportion of carcasses, $a$, that have landed in searched areas of variable configuration. Conditioned on a given carcass realization, $a$ is a parameter. Several methods have been proposed to estimate $a$ (Jain 2005; Huso and Dalthorp 2014; Good et al. 2011; Studyvin et al. 2019). A fourth method used by, for example, Gruver et al. (2009) and Drake et al. (2012) calculates $a$ as the simple proportion of area searched withing the designated plot. This assumes that the designated plot comprises all areas within which a carcass could fall and the carcass density is independent of distance, "an implausible assumption when turbineinduced mortality occurs" (Huso and Dalthorp 2014). We compared the first three methods, but the method used by Studyvin et al. (2019) had not been published when we completed our analysis and is not included.

The methods described in this section assume data are derived from carcass surveys conducted on plots that extend at most $r_{\max } \mathrm{m}$ from the center of the turbines selected for surveying. Let $i$ in $\{1, \ldots, C\}$ index each observed carcass with $C$ as the total number of observed carcasses. Each method can be implemented by recording the location of each carcass as a distance, $r_{i}$, and direction, $\theta_{i}$ in $[0,2 \pi)$, with respect to the nearest turbine. Note that we assume no carcass can land within $2 \mathrm{~m}$ of the center of any turbine due to the girth of the tower; therefore, $r_{i}$ is in $\left[2, r_{\max }\right]$.

In the description of the methods in this section, we assume that the searched area has been mapped out in a GIS software, so that information such as the proportion or amount of area searched at a specified distance or location relative to nearest turbines is known.

In the description of some methods below, and in the results presented in Sect. 4, we assume the conditional probability, $G$, that a carcass is observed given it landed on $\mathrm{R} \& \mathrm{P}$ is known. In addition, for the ratio method, we assume the probability, $G_{c l r}$, that a carcass is observed given it landed on non-R\&P at a turbine cleared of vegetation and surveyed is also known. In practice, these quantities are unknown, and numerous methods have been proposed for their estimation (Shoenfeld 2004; Huso 2011; KornerNievergelt et al. 2015; Wolpert 2015; Dalthorp et al. 2017). Including uncertainty in mortality estimates from the probability of detection is beyond the scope of this 
research. For the comparison of all methods, except the ratio method, it is acceptable to assume $G$ is known, because $a$ is estimated independently of $G$, so uncertainty from estimating $G$ would have a similar effect on these methods. In the case of the ratio method, the detection probabilities in the two visibility classes are used to estimate $a$, so assuming these values are known results in more precise estimates of $a$ than would be observed in practice; however, as discussed in Sect. 4, the performance of this method is generally poor, so this slight advantage does not obscure the conclusions of this research.

In this section we introduce five methods for obtaining a point estimate for $a$. In Sect. 5 we propose a general method that can be used to calculate a standard error of $\hat{a}$ or confidence interval for $a$ using any of the methods described below.

\subsection{Cake method}

Jain (2005) proposed accounting for unsearched areas by partitioning the area beneath turbines into $10 \mathrm{~m}$ concentric annuli, or rings, and calculating mortality independently for each ring. This method intrinsically assumes that carcass density is homogeneous within an annulus, which under isotropic carcass spatial distributions, becomes more reasonable as the buffer-distance between the outer and inner radii of a ring-goes to zero. In practice, the lower bound of the buffer will be determined by the resolution of the equipment used to record carcass distance with respect to the center of the nearest turbine. The Cake method refers to the methodology proposed by Jain (2005) with a buffer $<10 \mathrm{~m}$. We introduce the notation of the Cake method in this section, and examine the relative performance of this methodology in the results presented in Sect. 4 utilizing a $1 \mathrm{~m}$ buffer.

To implement the Cake method, let $j$ in $\left\{2, \ldots,\left(r_{\max }-1\right)\right\}$ index the rings, $C_{j}=$ $\sum_{i=1}^{C} \mathrm{I}\left(r_{i}=j\right)$ be the number of observed carcasses $j \mathrm{~m}$ from the center of the surveyed turbines, and $A_{j}$ be the known proportion of area searched $j \mathrm{~m}$ from the surveyed turbines. For an individual ring, the total number of fatalities, $M_{j}$, is estimated as,

$$
\hat{M}_{j}=\frac{C_{j} / G}{A_{j}}
$$

This method directly estimates $M$ with $\widehat{M}_{c a k e}=\sum_{j=2}^{\left(r_{\max }-1\right)} \hat{M}_{j}$. Given $\widehat{M}_{\text {cake }}$, the estimate of $a$ is calculated as

$$
\hat{a}_{\text {cake }}=\frac{C / G}{\widehat{M}_{\text {cake }}}=\frac{C}{\sum_{j=2}^{\left(r_{\max }-1\right)} \frac{C_{j}}{A_{j}}} .
$$

We refer to this as the "Cake" method, because annuli closest to the turbine tend to have the highest density of carcasses (Fig. 1), and the assumption of homogeneity within an annulus creates a step function yielding a tiered wedding cake appearance when plotted in three dimensions. 


\subsection{Ratio method}

Good et al. (2011) used ratio estimation (Thompson 2012) at Fowler Ridge Wind Farm in Indiana, USA to account for unsearched areas. The R\&P of 109 out of 355 turbines were searched out to $40 \mathrm{~m}$, and in addition, a square $80 \times 80 \mathrm{~m}$ plot around 9 of the turbines was cleared of vegetation and searched, albeit with a lower searcher efficiency than on the R\&P.

Implementation of the ratio method requires each carcass be assigned an indicator, $\mathrm{I}(\mathrm{clr})_{i}$, to indicate if the carcass was found at a turbine cleared of vegetation (regardless of visibility class), and an indicator, $\mathrm{I}(R P)_{i}$, to indicate if the carcass was observed on R\&P. This information can be obtained either using the location data, or within survey records.

To implement this method, define $C_{R P}=\sum_{i=1}^{C}\left(\mathrm{I}(\mathrm{clr})_{i} \cdot \mathrm{I}(R P)_{i}\right)$ as the number of observed carcasses on R\&P at turbines cleared of vegetation, and $C_{n o n R P}=$ $\sum_{i=1}^{C}\left(\mathrm{I}(c l r)_{i} \cdot\left(1-\mathrm{I}(R P)_{i}\right)\right)$ as the number of observed carcasses on non-R\&P at turbines cleared of vegetation; and recall that $G$ and $G_{c l r}$ are the known conditional probabilities a carcass is observed given it landed on R\&P or non-R\&P, respectively, at surveyed turbines. Implementing the ratio method, $a$ is estimated as

$$
\hat{a}_{\text {ratio }}=\frac{\frac{C_{R P}}{G}}{\frac{C_{R P}}{G}+\frac{C_{\text {non } R P}}{G_{c l r}}} .
$$

When applying the ratio method in the simulations described in Sect. 3, we deviated from the protocol used by Good et al. (2011), simulating estimates from a protocol where the cleared plots encompass the entire spatial carcass distribution (out to $120 \mathrm{~m}$ ), and the R\&P are searched out to $120 \mathrm{~m}$ at every turbine. Obviously this would produce more precise and accurate estimates relative to cleared plots that do not comprise the full spatial carcass distribution and R\&P searches conducted on less than one third of the total number of turbines.

\subsection{Weighted distribution (WD) method}

The weighted distribution method uses maximum likelihood estimation to estimate the univariate carcass distance distribution by viewing the observed carcass distances, $\left\{r_{1}, \ldots, r_{c}\right\}$, as a random sample from the observed carcass distribution, $f_{\mathrm{o}}(r \mid \Theta)$. Assuming that carcass distance (both observed and unobserved) follows a parametric family distribution (e.g. Weibull, Gamma, Log-normal, etc.), indexed by a parameter vector $\Theta$, and the spatial carcass distribution is isotropic - independent of directionthe pdf of the observed carcass distribution is

$$
f_{\mathrm{o}}(r \mid \Theta)=\frac{A(r) f(r \mid \Theta)}{\int_{2}^{r_{\text {max }}} A(r) f(r \mid \Theta) \mathrm{d} r} \mathrm{I}\left(r \in\left[2, r_{\text {max }}\right]\right),
$$

where $A(r)$ is a known function that yields the fraction of area searched $r$ meters from the center of surveyed the turbines (Patil and Rao 1978). Heuristically, this assumes 


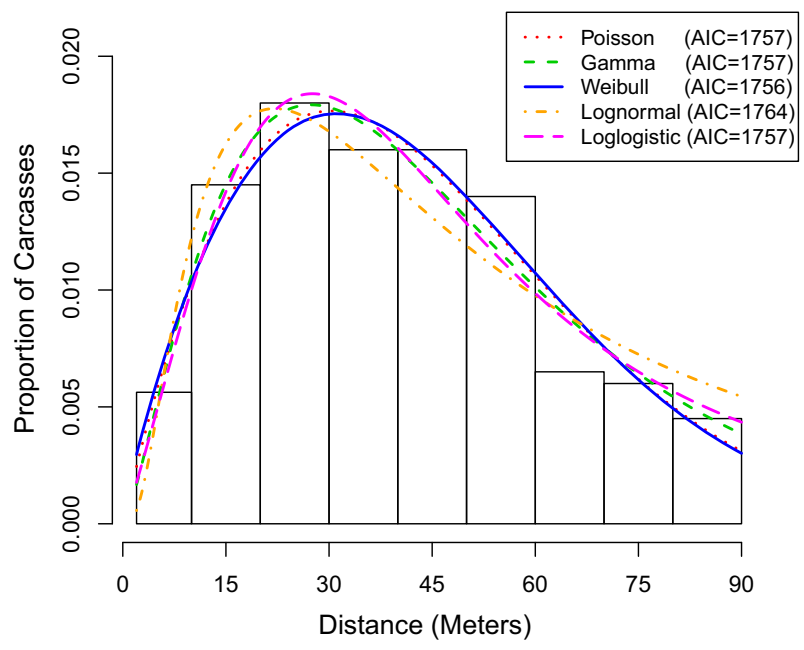

Fig. 2 Histogram of the 200 observed carcasses distances described in Fig. 1 Left, along with fitted truncated probability density functions from select parametric distributions

the probability a carcass is found at a given distance is proportional to the probability a carcass lands at that distance, times the proportion of that distance searched. The maximum likelihood estimate ( $m l e)$ of $\Theta, \hat{\Theta}_{m l e}$, can be estimated by maximizing the log-likelihood function,

$l\left(\Theta \mid\left\{r_{1}, \ldots, r_{c}\right\}\right)=\sum_{i=1}^{C} \log \left(A\left(r_{i}\right)\right)+\log \left(f\left(r_{i} \mid \Theta\right)\right)-\left[\log \left(\int_{2}^{r_{\max }} A(r) f\left(r_{i} \mid \Theta\right) \mathrm{d} r\right)\right]$

which can be used to estimate $a$ as

$$
\hat{a}_{w d}=\int_{2}^{r_{\max }} A(r) f\left(r \mid \hat{\Theta}_{m l e}\right) \mathrm{d} r
$$

In practice, this method can accommodate any parametric family distribution, and AIC (Akaike 1998) can be used to compare between fitted models. In obtaining the results discussed Sect. 3, we assumed carcass distance can be modeled with a Weibull distribution, because it produced the lowest AIC of the parametric models considered for modeling the distance of the carcass distances (Fig. 2). We also assumed distance to the nearest turbine could be measured within a meter, and recorded $r_{i}$ as either $2.5,3.5, \ldots$, or 119.5 . We defined $A(r)$ as the proportion of area within 0.5 meters of $r$ contained in R\&P, which is analogous to $A_{(r-0.5)}$ in the notation introduced for the Cake method.

After development, we shared this method with researchers who implemented the weighted distribution method in the context of estimating eagle fatalities at wind projects (Hallingstad et al. 2018). 


\subsection{Generalized linear model method}

Originally proposed by Arnett et al. (2009), Huso and Dalthorp (2014) examined the statistical properties of accounting for unsearched areas by partitioning the area beneath surveyed turbines into a grid and modeling the spatial distribution of carcasses with a generalized linear model $(\mathrm{glm})$.

Huso and Dalthorp (2014) introduced their method using logistic regression. Warton and Shepherd (2010) demonstrated that a Poisson point process model produces similar coefficient estimates and standard errors as a logistic regression as long as the grid used to fit the models is sufficiently dense. In the simulations described in Sect. 3, we used a $1 \mathrm{~m}$ dense grid and results for the logistic and Poisson regression models were virtually indistinguishable. The Poisson model is advantageous in this setting as it allows for more than one carcass to occupy a grid cell. It is worth noting that this method can also be established by partitioning the area beneath turbines into rings, similar to the Cake method. We chose to use grid notation to draw attention to the similarity of this method to the Anisotropic glm method introduced below.

The method to account for unsearched areas developed by Huso and Dalthorp (2014) in the context of a Poisson regression model fit on a grid where cells are indexed by distance, $r$ in $\left[2, r_{\max }\right] \mathrm{m}$, and direction, $\theta$ in $[0,2 \pi)$ radians, to the nearest turbine, assumes the number of observed carcasses in a grid cell, $C_{r, \theta}=$ $\sum_{i=1}^{C}\left(\mathrm{I}\left(r=r_{i}\right) \cdot \mathrm{I}\left(\theta=\theta_{i}\right)\right)$, follows a Poisson distribution. Its mean, $\lambda_{r, \theta}$, modeled as a function of distance from the turbine,

$$
C_{r, \theta} \sim \operatorname{Poisson}\left(\lambda_{r, \theta}\right) \quad \log \left(\lambda_{r, \theta}\right)=\beta_{0}+\beta_{1} r+\beta_{2} r^{2}+\log \left(O_{r, \theta}\right),
$$

where $O_{r, \theta}$ is the offset for the cell indexed by $(r, \theta)$, and is equal to the number of turbines at which that cell is searched. The fitted Poisson regression model can be utilized to estimate the spatial carcass distribution, $\hat{f}(r, \theta)$, as

$$
\hat{f}(r, \theta)=\frac{\exp \left(\hat{\beta}_{0}+\hat{\beta}_{1} r+\hat{\beta}_{2} r^{2}\right)}{\int_{0}^{2 \pi} \int_{2}^{r_{\max }} \exp \left(\hat{\beta}_{0}+\hat{\beta}_{1} r+\hat{\beta}_{2} r^{2}\right) r \mathrm{~d} r \mathrm{~d} \theta} \mathrm{I}\left(r \in\left[2, r_{\max }\right]\right) .
$$

The estimate of $a$ is obtained by integrating $\hat{f}(r, \theta)$ over the searched area. If an $s$ meter dense grid is utilized, with $\mathcal{S}$ representing the collection of points indexed by $(r, \theta)$, then the integral can be calculated numerically as

$$
\hat{a}_{g l m}=\sum_{(r, \theta) \in \mathcal{S}} \hat{f}(r, \theta) \frac{s^{2} O_{r, \theta}}{T},
$$

where $T$ is the total number of turbines at the wind facility. 


\subsection{Anisotropic generalized linear model method}

The cake, weighted distribution, and $g l m$ methods all assume the spatial carcass distribution is isotropic. Under an anisotropic distribution, carcass density changes with direction, in addition to distance, with respect to the nearest turbine. The $\mathrm{glm}$ method can readily be modified to account for anisotropic carcass distributions by incorporating direction, $\theta$, into the Poisson regression model. Thus, the Anisotropic glm method, like the $\mathrm{glm}$ method also partitions the area beneath surveyed turbines into a grid with cells indexed by $r$ in $\left[2, r_{\text {max }}\right] \mathrm{m}$ and $\theta$ in $[0,2 \pi)$ radians, and assumes that the number of observed carcasses for a specific grid cell, $C_{r, \theta}=\sum_{i=1}^{C}\left(I\left(r=r_{i}\right) \cdot I\left(\theta=\theta_{i}\right)\right)$, follows a Poisson distribution; however, the anisotropic glm allows $\mathrm{E}\left(C_{r, \theta}\right)=\lambda_{r, \theta}$ to differ by direction.

Direction has to be included into the model as a circular variable, which accounts for the cyclic pattern of direction in which, for example, $0^{\circ}$ and $360^{\circ}$ are equivalent and $5^{\circ}$ and $355^{\circ}$ are equidistant from $0^{\circ}$. There are numerous techniques for incorporating circular variables into regression models (see Pewsey et al. (2013)). Perhaps the simplest approach for modeling a continuous response, $Y$, as a function of a circular variable, $\theta$, is $\mathrm{E}(Y)=\beta_{0}+\beta_{1} \cos (\theta)+\beta_{2} \sin (\theta)$. This model can be made more flexible by considering the $K$ th Fourier expansion on $\theta$, and including cosine and sine terms for each of $k \theta, k \in\{1, \ldots, K\}$. Accounting for an anisotropic spatial carcass distribution in the context of Poisson regression requires allowing the predicted carcass density for a given distance to differ by direction, which can be achieved using interactions between distance terms and direction terms (represented by sine and cosine terms),

$$
\begin{aligned}
\log \left(\lambda_{r, \theta}\right) & =\beta_{0}+\beta_{1} r+\beta_{2} r^{2} \\
& +\sum_{k=1}^{K} \beta_{4 k-1} r \cos (k \theta)+\beta_{4 k} r \sin (k \theta)+\beta_{4 k+1} r^{2} \cos (k \theta)+\beta_{4 k+2} r^{2} \sin (k \theta) \\
& +\log \left(O_{r, \theta}\right) .
\end{aligned}
$$

The main effects for angle (i.e. $\sin (\theta)$ and $\cos (\theta))$ are not incorporated into (5), because it seems reasonable to assume a common intercept. In this application, no carcass can land within $2 \mathrm{~m}$ of the turbine, but theoretically the generalized linear model used to estimate the spatial carcass distribution produces an estimated carcass density at the origin. If the main effects for angle were included in the model, the estimated density at the origin could change depending on the value for $\theta$ that is used, providing multiple density estimates for a single location. To avoid this issue, the main effects are not included in (5).

The value of $K$ represents the order of the Fourier expansion on $\theta$. Higher values of $K$ allow for more flexible models; however, increasing $K$ by 1 requires estimation of four additional parameters. An excess of terms in the model could potentially increase the variation in mortality estimates. In practice, different values of $K$ can be considered, along with nested models, such as those excluding sine terms or terms with $r^{2}$, and the appropriate Poisson regression model can be obtained using any reasonable model selection method. To obtain the Poisson regression model for the anisotropic $\mathrm{glm}$ method in the simulation described in Sect. 3, we used forward model selection, 
increasing the value of $K$ one unit at a time (up to $K=3$ ), and using the likelihood ratio test with $\alpha=0.05$ to compare models.

The anisotropic $\mathrm{glm}$ method differs from the (isotropic) $\mathrm{glm}$ method only in the Poisson regression model used to obtain the estimated bivariate carcass distribution. After obtaining the fitted model, the procedure for estimating $a$ is identical. The fitted values from anisotropic method, $\widehat{\log \left(\lambda_{r, \theta}\right)}$, can be used to model the anisotropic spatial carcass distribution (designated by superscript (a)) as

$$
\hat{f}^{(a)}(r, \theta)=\frac{e^{\widehat{\log \left(\lambda_{r, \theta}\right)}}}{\int_{0}^{2 \pi} \int_{2}^{r_{\text {max }}} e^{\widehat{\log \left(\lambda_{r, \theta}\right)}} r \mathrm{~d} r \mathrm{~d} \theta} \mathrm{I}\left(r \in\left[2, r_{\text {max }}\right]\right) .
$$

Analogous to (4), if an $s$-meter dense grid is utilized, with $\mathcal{S}$ representing the collection of points indexed by $(r, \theta)$, the estimate of $a$ is calculated as

$$
\hat{a}_{g l m}^{(a)}=\sum_{(r, \theta) \in \mathcal{S}} \hat{f}^{(a)}(r, \theta) \frac{s^{2} O_{r, \theta}}{T} .
$$

\section{Simulation study}

We compared the performance of the five methods described above using simulations designed to (i) evaluate accuracy and precision of estimates for the proportion of carcasses that land in the searched area, i.e., on R\&P; (ii) evaluate the influence of various conditions, e.g., per-turbine fatality rate, $\mathrm{R} \& \mathrm{P}$ configuration, spatial density patterns, etc. on the accuracy and precision of each method; and (iii) understand how variability in $\hat{a}$ contributes to variation in mortality estimates, $\hat{M}$. The simulations considered four spatial carcass distributions, two R\&P configurations of actual wind projects, and four per-turbine fatality rates. One thousand trials were performed for every combination of settings. Each trial simulated a data set of observed carcasses, designed to mimic the random process that would be observed in practice.

\subsection{Distance distributions}

Our capacity to accurately estimate the fraction of carcasses that land within our search boundaries depends on whether the spatial distribution is isotropic and if not, whether our search boundaries comprise a disproportionate amount of area with either higher or lower carcass density. Actual spatial distributions of carcasses will likely depend on (i) wind speed at the time of collision that will affect distance the carcass travels downwind, (ii) location of the collision along the blade that will determine the relative speed at which the blade is moving when the carcass is struck, and (iii) position of the blade in its rotational arc that will determine whether the carcass is lifted as it is struck or forced directly downward, as well as turbine height, topography, scavenger behavior, and other factors. 

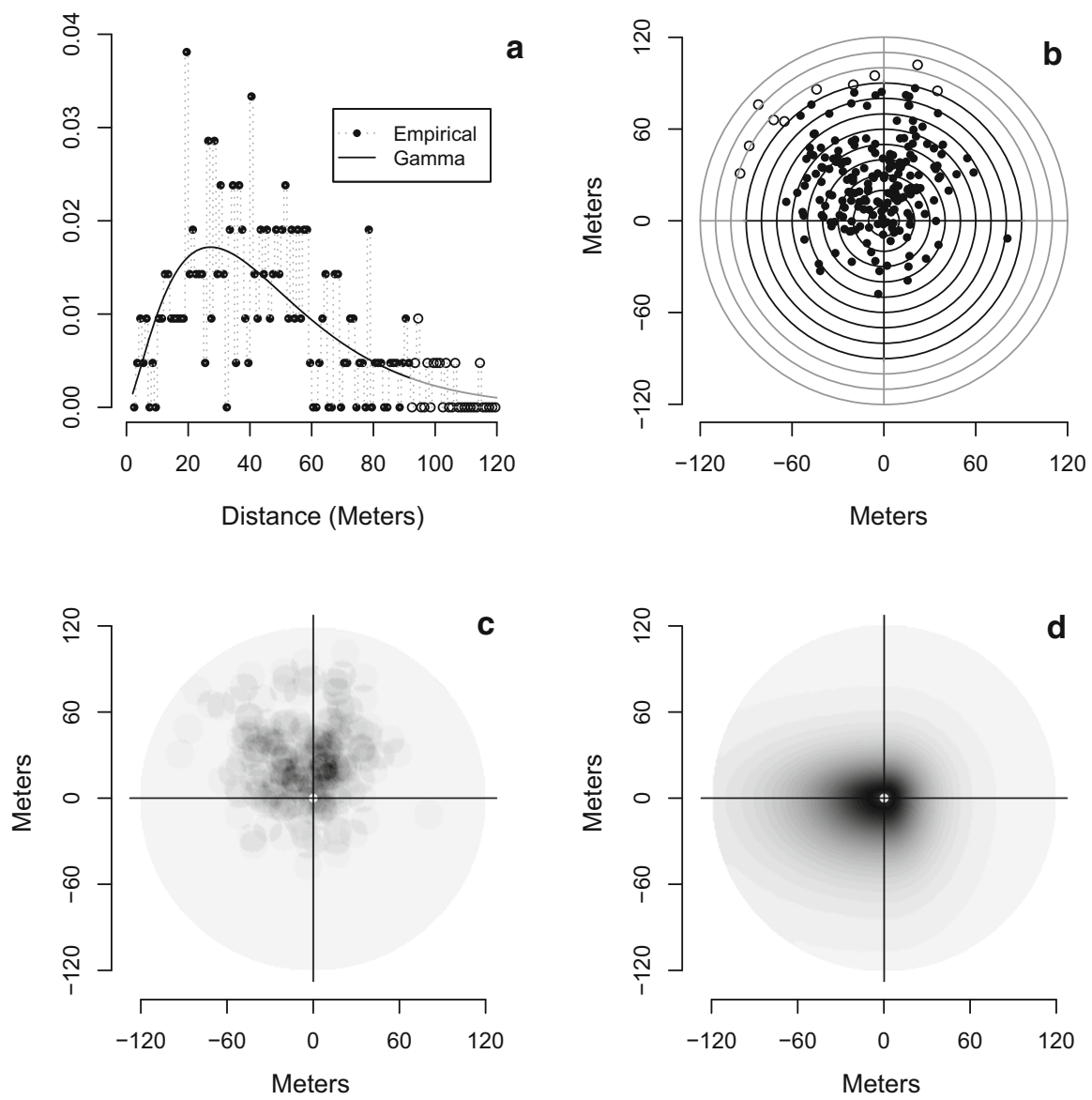

Fig. 3 Underlying carcass distributions used to simulate carcass locations. Created using 200 observed carcass locations displayed in Fig 1 Left. a Isotropic Empirical (dots) and Gamma (smooth line) distributions created using observed distance of each carcass to the nearest turbine (open points indicate simulated data.) b Carcass location relative to the nearest turbine, oriented relative to a southern originating average nightly wind direction (open points indicate simulated data.) c Heatmap of the Anisotropic Empirical distribution created by sampling the locations in (b) and uniformly shifting within a $10 \mathrm{~m}$ radius. d Heatmap of the Anisotropic Gamma distribution

We simulated carcass locations using four spatial distributions from factorial combinations of isotropy/anisotropy and empirical/modeled based on 200 bat carcasses observed at a wind project in the Midwestern U.S. from April to November in 2013 (Figs. 1, 2, and 3). Searches were conducted daily on 15 circular plots cleared of vegetation out to $90 \mathrm{~m}$ from the turbine. Assuming constant probability of observing a carcass within each cleared plot, the distribution of observed carcasses provides an estimate for the true underlying spatial carcass distribution. Furthermore, searchers were able to determine that the 200 carcasses arrived the night prior to the search, enabling us to assign covariate information, such as average nightly wind direction, which was used to create an anisotropic spatial carcass distribution. 
We refer to the two spatial carcass distributions that are independent of direction as the Isotropic Empirical distribution and the Isotropic Gamma distribution (Fig. 3a). The Isotropic Empirical distribution is the empirical distribution of the 200 observed carcass distances to the nearest meter, plus 10 simulated carcass distances. The simulated carcass distances were included because the empirical distribution (and corresponding parametric fits) indicated that carcasses likely landed past $90 \mathrm{~m}$ (Fig. 2). Therefore we used the triangular distribution on $90 \mathrm{~m}$ to $120 \mathrm{~m}$, to simulate ten additional locations increasing the total by $5 \%$ to create a more realistic carcass distribution. The Isotropic Gamma parameters, shape $=2.4$ and scale $=19.5$, were calculated by fitting a Gamma distribution to the observed carcass distances, and then using this fit to extrapolate to $120 \mathrm{~m}$. The Gamma distribution was utilized, because it produced the third lowest AIC of the parametric methods we considered to model carcass distance (Fig. 2). Although the Weibull distribution and scaled-Poisson regression model had lower AICs, we chose to model the spatial distribution with the Gamma to avoid favoring either the weighted distribution (3), or the $\operatorname{glm}$ (4), methods by generating and fitting data with the same model.

We refer to the two spatial carcass distributions that depend on direction as the Anisotropic Empirical distribution and the Anisotropic Gamma distribution. The Anisotropic Empirical distribution was created using the location of the 200 observed carcasses, maintaining their distances but rotating them by an amount equal to the average wind direction on the night they were killed minus $180^{\circ}$. This simulates the location relative to a prevailing wind originating from the south (Fig. 3b). To these, we added the same 10 simulated distances used for the Isotropic Empirical distribution, and the angle was determined by sampling with replacement from the rotated angles that landed at least $45 \mathrm{~m}$ from the turbine. Simulated carcass location under the Anisotropic Empirical distribution was assigned by randomly sampling a location displayed in Fig. $3 \mathrm{~b}$ and shifting the coordinates uniformly within a 10 -m radius of the sampled location (Fig. 3c). The 10-m shift represents a compromise between reproducing the observed data too closely vs. not closely enough. The Anisotropic Gamma distribution (Fig. 3d) was created by distorting the spatial carcass distribution of the Isotropic Gamma distribution, to create a realistic anisotropic distribution, while maintaining the same univariate carcass distance distribution to its isotropic counterpart. The Anisotropic Gamma distribution simulates conditions where the prevailing winds originate in the east; carcasses are twice as likely to land west (downwind) of the turbine, where they are also more dispersed relative to carcasses that land east (upwind) of the turbine. There is a vast variety of possible patterns of anisotropy, and it is well beyond the scope of this study to analyze or characterize them all in any meaningful way. Instead, we chose two contrasting patterns that represent two somewhat extreme cases because the wind directions in each are so constant, as illustrated in the contrast between the observed carcass dispersion pattern (Fig. 1a) with variable winds and the anisotropic dispersion pattern derived from the same data but adjusted to mimic a constant prevailing wind direction (Fig. 3b).

To simulate carcass location for a specific spatial carcass distribution, we assigned a probability mass to each $1 \mathrm{~m}$ grid cell, and sampled grid cells proportionally to the probability a carcass lands in that cell. For more detail see the R code in Appendix 1. 


\subsection{R\&P configurations}

We selected two current wind projects to demonstrate how the methods perform under completely different R\&P configurations. WP1 is located amid corn and soy farms in Indiana, has 355 turbines, and road orientation is predominately aligned with cardinal directions. WP2 is located along two mountain ridges in Pennsylvania and has 23 turbines. The roads at WP2 have more twist and turns with no pronounced unifying pattern like WP1. The R\&P configurations for both facilities were obtained using Google Earth, mapped in ArcGIS, and imported into R R-statistical software (R Core Team 2015) as polygons (Fig. 4).

\subsection{Average number of fatalities per turbine}

In a given simulation trial, mortality was determined by the number of turbines at the wind project, 355 at WP1 and 23 at WP2, and the average number of carcasses per turbine: $2,10,25$, or 70 . We chose these mortality levels to represent the range of rates reported in the publicly available literature. A rate of 70 carcasses per turbine represents the average of two of the highest estimated mortality per turbine rates we are aware of. BHE Environmental (2011) estimated 50.5 (90\% CI 36.1-70.3) bat fatalities per turbine at Cedar Ridge Wind Farm in Wisconsin, and Hein et al. (2014) estimated 90 (95\% CI 57-153) fatalities per fully operational turbine at Pinnacle Wind Farm in West Virginia. We chose 2 carcasses per turbine to evaluate how the methods preform with few observed carcasses, while trying to avoid generating trials with 0 observed carcasses at WP2, as such trials provide no information on how the methods compare to each other and complicate the interpretation of the results if excluded. With a rate of 2 carcasses per turbine, the average number of observed carcasses per trial at WP2 is about 8 for each carcass distribution (Table 1), and the probability that 0 carcasses are observed is less than 0.00015 . Averages of 10 and 25 carcasses per turbine were selected to assess the performance of the methods when the rate is between 2 and 70 .

While mortality was fixed for a set of conditions, the number of observed carcasses differed in each trial. The expected number of carcasses observed depends on spatial carcass distribution, $\mathrm{R} \& \mathrm{P}$ configuration, and average number of carcasses per turbine (Table 1).

\subsection{Simulating carcass counts}

Simulations were designed to mimic a setting where R\&P carcass surveys were conducted at every turbine, to the maximum assignable distance from the turbine: 120 $\mathrm{m}$. To simulate a set of observed carcasses used to estimate mortality, $M$ simulated carcasses were assigned (i) a turbine number using the discrete uniform distribution on the number of turbines at the wind project, (ii) a carcass location using one of the four spatial carcass distributions, (iii) an indicator of whether the carcass landed on an R\&P based on its location and the specific R\&P configuration of its turbine, and (iv) an indicator of whether the carcass was observed based on a random draw from a 


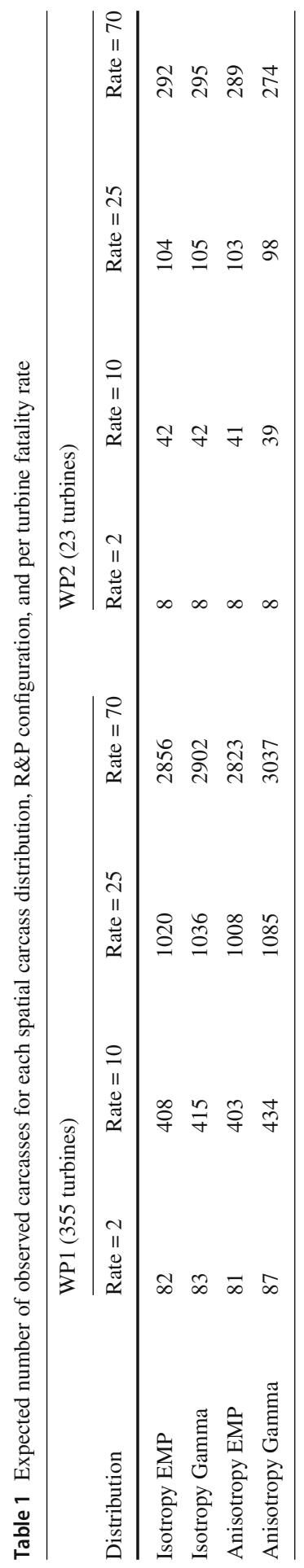


Bernoulli with $p=0.85$ (Good et al. 2011). In each trial $M$ is a fixed value equal to the number of turbines times the mean fatality per turbine rate.

In addition to $\mathrm{R} \& \mathrm{P}$ surveys, the ratio method also requires information from clearedplot surveys. Good et al. (2011) searched 9 out of 355 (2.5\%) turbines cleared of vegetation within an $80 \mathrm{~m} \times 80 \mathrm{~m}$ plot centered at the turbine to provide information for the ratio estimator. To reflect this method, we simulated carcass surveys on non$\mathrm{R} \& \mathrm{P}$ areas within $120 \mathrm{~m}$ of 9 turbines for WP1, although, this represents a far greater area than was searched in practice. We are not aware of the ratio method having been applied at facilities with only 23 turbines. Clearing the same percent of turbines at WP2 as WP1 corresponds to clearing $<1$ turbine; therefore, we arbitrarily chose to conduct simulated surveys on non-R\&P at 3 out of $23(13 \%)$ turbines. The turbines selected for clearing differed in each trial, and were selected using a simple random sample. Each carcass on non-R\&P was assigned an indicator of whether it was observed based on a random draw from a Bernoulli with $p=0.32$ as reported by Good et al. (2011).

Simulations were perfomed using R-statistical software. One thousand trials were generated for every combination of settings ( 2 R\&P configurations $\times 4$ distributions $\times$ 4 rates $=32$ combinations). We applied all five methods to the same data generated in every trial, or simulated data set, to obtain the estimated proportion, $\hat{a}$, of carcasses that land on R\&P for each combination of settings, except at WP2 where the anisotropic $\mathrm{glm}$ method was not applied when the rate was 2 due to the low number of simulated carcasses observed (Table 1).

\section{Results}

The objective of this research is to compare several methods used to estimate $a$; however, in practice, $a$ is a nuisance parameter that must be estimated in order to estimate the true quantity of interest: mortality, $M$. Therefore, in addition to displaying and discussing each method's performance in estimating $a$ (Figs. 5 and 6), we show how variability in $\hat{a}$ contributes to variability in mortality estimates by considering mortality estimate,

$$
\hat{M}=\frac{C}{\hat{a} \cdot G},
$$

where $\mathrm{C}$ was the number of observed carcasses on $\mathrm{R} \& \mathrm{P}$ and $G$ is the known probability a carcass is observed given it landed within the searched area (Table 2). In practice $G$ is unknown and must be estimated, in which case the estimator $\hat{M}$ would involve $\hat{G}$.

In displaying the results of $\hat{a}$ for each method, we calculated $\frac{\hat{a}}{a}$ for each estimate in every trial. We divided $\hat{a}$ by $a$, because $a$ is a random variable that changes from trial to trial even under the same settings (e.g. underlying carcass distribution, R\&P configurations, and fatality per turbine rate). We chose to plot the results on the log scale, because underestimating $a$ leads to greater bias in mortality estimates relative to an equidistant overestimate of $a$. 


\subsection{Isotropic spatial carcass distributions}

In general, each method performed at its relative best at both wind projects under isotropic spatial carcass distributions with high average fatality rates per turbine (Table 2, Figs. 5 and 6). The cake, weighted distribution, $g l m$, and anisotropic glm methods tend to produce similar results for estimates of mortality, $(\hat{M})$, with the anisotropic glm method having a slightly larger standard deviation in some cases. The maximum observed bias for these methods under isotropic distributions with an average fatality per turbine rate of 70 is $3.9 \%$. The ratio method produces the most biased results and the largest standard deviation in almost all cases. Under the Isotropic Empirical distribution, when the average rate of carcasses per turbine is 70, the standard deviation of mortality estimates for the ratio method at WP1 and WP2 are 3.5 and 1.5 times larger, respectively, than the standard deviation of the other four methods. To prevent its large variation from obscuring results of other estimators, we do not include results from the ratio estimator in Figs. 5 or 6, but the results are given in Table 2.

As expected, as the rate of carcasses per turbine decreases, the number of observed carcasses decreases and the variation of $\frac{\hat{a}}{a}$ increases for all methods. For the ratio method, both bias and the variation of $\hat{a}$, and consequently $\hat{M}$, increase as the average rate of carcasses per turbine decreases. For example, under the Isotropic Empirical distribution at WP1 the relative bias of the ratio method increases from $2.4 \%$ when the average rate is 70 to $15.5 \%$ when the rate is 2 (Table 2). In addition, the ratio method cannot produce a mortality estimate if 0 carcasses are observed on the R\&P at cleared turbines. When the mortality rate was low (2/turbine) this occurred in about $10 \%$ of trials at WP1 and more than $33 \%$ of trials at WP2, for every spatial carcass distribution. Results for which more than $1 / 3$ of the estimates are inestimable are reported as NR.

\subsection{Anisotropic spatial carcass distributions}

The effect that anisotropic spatial carcass distributions have on each of the methods differed by wind project and the two anisotropic distributions. Consider the results from the cake, weighted distribution, and isotropic glm methods. Again, these methods all performed similarly for both anisotropic distributions at both wind project R\&P configurations (Table 2, Figs. 5 and 6). It is not surprising that these methods are affected similarly in each setting, because none of the methods take direction into consideration in addition to distance, and therefore intrinsically assume the spatial carcass distribution is isotropic. The results from the simulations suggest that when this assumption is violated the effect depends on the spatial carcass distribution, and R\&P configurations. Under the Anisotropic Empirical distribution, these methods overestimate $a$ and consequently underestimate mortality at WP1 and WP2; while under the Anisotropic Gamma distribution, these methods overestimate mortality at WP1 and underestimate mortality at WP2 (Figs. 5 and 6).

The direction of the bias when estimating mortality using a method that assumes isotropy can be explained by examining the anisotropic distribution and a composition plot of the R\&P configurations at the wind project of interest (Fig. 7). A composition plot displays the proportion of turbines' $\mathrm{R} \& \mathrm{P}$ that contain a specific point. Points 


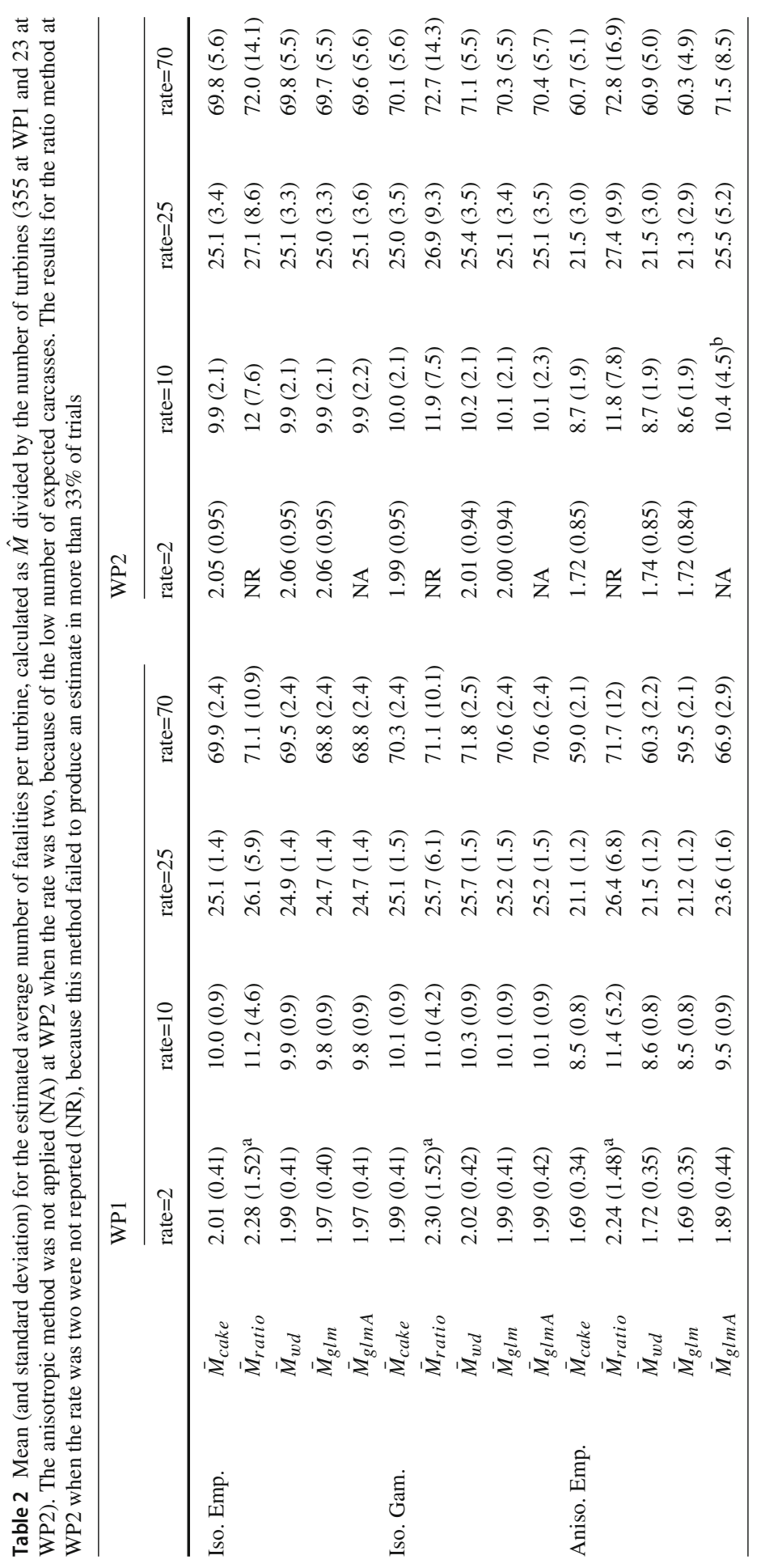




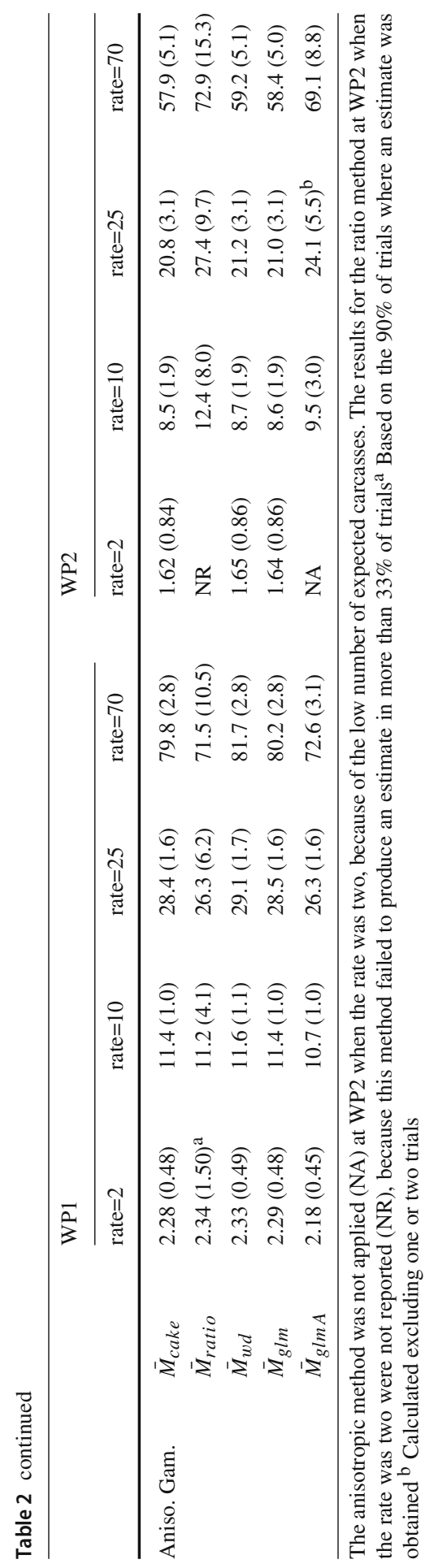


that are darker are contained in the R\&P of many turbines, and points that are lighter are contained in the R\&P on few turbines. From this plot it becomes apparent that most of the roads at WP1 are aligned with cardinal directions. Furthermore, most roads approach the turbine from the west, and fewest from the north. Now consider the two anisotropic distributions displayed in Fig. 3. Recall, under the Anisotropic Gamma carcass distribution, most carcasses land west of the turbine; and given the $\mathrm{R} \& \mathrm{P}$ configuration at WP1, the most data for any distance greater than $10 \mathrm{~m}$ from the turbine is collected west of the turbine. This causes the isotropic methods to overestimate the number of carcasses at most distances, producing an overestimate of mortality. Under the Anisotropic Empirical distribution relatively few carcasses land west of the turbine producing an underestimate of mortality. Using the same logic and Fig. 7(right), the direction of the bias for mortality estimates at WP2 using an isotropic method can be explained.

Three trials were excluded when calculating the results for the anisotropic $\mathrm{glm}$ method at WP2: two under the Anisotropic Empirical distribution when the rate was 10, and one under the Anisotropic Gamma distribution when the average rate was 25. In each of these trials, the forward selection method identified the Poisson regression model with the third order Fourier expansion of $\theta$ as the most appropriate model, and the estimated mortality exceeded 4000 carcasses per turbine. These unreasonable estimates occur when the quadratic term for distance, which is the sum of seven terms for a fixed direction (see Eq. 5), is positive, causing predicted carcass density to increase with distance from the turbine. Researchers can easily identify this situation in practice with a graphical display, and use a lower order Fourier expansion or avoid this anisotropic method altogether; therefore, it is reasonable to exclude these trials when reporting the results. Attention to these trials is included here, so researchers are aware of a potential issue that may arise when including quadratic terms in the generalized linear model.

The Anisotropic $\mathrm{glm}$ method had success in reducing the bias in mortality estimates under anisotropic spatial carcass distributions. When compared to the isotropic $\mathrm{glm}$ method, under the Anisotropic Empirical distribution the bias in $\hat{a}$ was reduced by $52 \%$ and $75 \%$ at WP1 for average rates of 2 and 70 respectively, with negligible increases in the standard deviation. Under the same distribution at WP2 the bias of $\hat{a}$ was reduced by $73 \%$ and $94 \%$ for average rates 10 and 70 respectively; however, there was a $31 \%$ and $16 \%$ increase in standard deviation.

Recall, the order of the Fourier expansion, $K$, was determined using forward selection by increasing $K$ one unit at a time and using the likelihood ratio test with $\alpha=0.05$ to compare models. Under the isotropic models, the coefficients for terms with cos and sin are all zero, and expectedly about $95 \%$ of the trials select the Poisson regression model with $K=0$, which is the model used in the isotropic glm method (Table 3). In general, under the anisotropic distributions, the anisotropic $\mathrm{glm}$ method routinely identified Poisson regression models with $K>0$.

The bias of the ratio method was relatively unaffected by the anisotropy at WP1 (Table 2). At WP2, there was an increase in the standard deviation of the ratio method under the anisotropic distributions. This is likely attributed to an increase in variation in the proportion of carcasses landing on the $\mathrm{R} \& \mathrm{P}$ at individual turbines. The ratio method assumes the proportion is similar among turbines. Under anisotropy, this assumption 
Table 3 Proportion of trials in which the anisotropic $g l m$ method used the $k^{\text {th }}$ order Fourier expansion for estimation using a forward model selection process based on p-values $(\alpha=0.05)$ from the likelihood ratio test

\begin{tabular}{|c|c|c|c|c|c|c|c|c|}
\hline & \multirow[t]{2}{*}{ Rate $=$} & \multicolumn{4}{|l|}{ WP1 } & \multicolumn{3}{|l|}{ WP2 } \\
\hline & & 2 & 10 & 25 & 70 & 10 & 25 & 70 \\
\hline \multirow[t]{4}{*}{ Iso. Emp. } & $K=0$ & 0.944 & 0.957 & 0.952 & 0.950 & 0.939 & 0.950 & 0.952 \\
\hline & $K=1$ & 0.055 & 0.042 & 0.043 & 0.049 & 0.056 & 0.046 & 0.047 \\
\hline & $K=2$ & 0.001 & 0.001 & 0.005 & 0.001 & 0.004 & 0.003 & 0.001 \\
\hline & $K=3$ & 0.000 & 0.000 & 0.000 & 0.000 & 0.001 & 0.001 & 0.000 \\
\hline \multirow[t]{4}{*}{ Iso. Gam. } & $K=0$ & 0.927 & 0.946 & 0.932 & 0.933 & 0.907 & 0.923 & 0.929 \\
\hline & $K=1$ & 0.070 & 0.047 & 0.061 & 0.059 & 0.083 & 0.074 & 0.069 \\
\hline & $K=2$ & 0.002 & 0.007 & 0.007 & 0.006 & 0.008 & 0.003 & 0.002 \\
\hline & $K=3$ & 0.001 & 0.000 & 0.000 & 0.002 & 0.002 & 0.000 & 0.000 \\
\hline \multirow[t]{4}{*}{ Aniso. Emp. } & $K=0$ & 0.100 & 0.000 & 0.000 & 0.000 & 0.132 & 0.001 & 0.000 \\
\hline & $K=1$ & 0.826 & 0.840 & 0.703 & 0.366 & 0.760 & 0.899 & 0.829 \\
\hline & $K=2$ & 0.062 & 0.061 & 0.010 & 0.000 & 0.092 & 0.074 & 0.091 \\
\hline & $K=3$ & 0.012 & 0.099 & 0.287 & 0.634 & 0.016 & 0.026 & 0.080 \\
\hline \multirow[t]{4}{*}{ Aniso. Gam. } & $K=0$ & 0.413 & 0.001 & 0.000 & 0.000 & 0.729 & 0.460 & 0.057 \\
\hline & $K=1$ & 0.538 & 0.831 & 0.620 & 0.200 & 0.248 & 0.485 & 0.845 \\
\hline & $K=2$ & 0.047 & 0.149 & 0.314 & 0.495 & 0.020 & 0.051 & 0.085 \\
\hline & $K=3$ & 0.002 & 0.019 & 0.066 & 0.305 & 0.003 & 0.004 & 0.013 \\
\hline
\end{tabular}

becomes less tenable at wind projects with large variation in R\&P configurations at individual turbines.

\section{Accounting for the uncertainty of $\hat{a}$}

When $a$ is unknown and estimated using the methods described above, uncertainty induced by $\hat{a}$ must be taken into account when calculating a confidence interval for $M$. In this context, a confidence interval for $a$ and $M$ can be calculated using a semiparametric bootstrap (Efron and Gong 1983); however in verifying this, we discovered using a standard bootstrap_-drawing $C$ locations with replacement from the distribution of observed locations and applying one of (1) through (5) to simulate estimates of $a$-in conjunction with the percentile method yields intervals below the desired coverage. This occurs because such a method does not account for binomial uncertainty in $a$.

Recall, $a$ becomes a parameter after conditioning on a specific carcass realization that is generated from an underlying point process. Under this paradigm, $F$, the number of carcasses that land on $\mathrm{R} \& \mathrm{P}$, follows a $\operatorname{Binomial}\left(M, P_{a}\right)$ distribution, where $P_{a}$ is the probability a carcass lands on $\mathrm{R} \& \mathrm{P}$ for the underlying point process specific to a wind project during the period when searches are conducted. The methods discussed in the previous sections actually provide direct estimates for $P_{a}$, therein providing a 
reasonable point estimate for $a=F / M$, because $E(a)=P_{a}$. Both the uncertainty in estimating $P_{a}$ and binomial uncertainty in $F$ must be accounted for in calculating a confidence interval for $a$, which can be accomplished using a semi-parametric bootstrap to simulate the distribution of $(\hat{a}-a)$.

A single bootstrap is obtained by simulating $F_{b o o t}$ from a $\operatorname{Binomial}(\hat{M}, \hat{a})$ distribution. Given $F_{\text {boot }}$, a simulated number of observed carcasses, $C_{b o o t}$, is obtained by drawing from a $\operatorname{Binomial}\left(F_{b o o t}, G\right)$ distribution. Observed distances are simulated by drawing with replacement $C_{\text {boot }}$ locations from the set of locations at which fatalities were observed, and $\hat{a}_{\text {boot }}$ can be obtained by applying the method used to estimate $a$ to the simulated locations. A $(1-\alpha)$ confidence interval for $a$ is created by adding the $\alpha / 2$ and $1-\alpha / 2$ quantile from the distribution of $\left(\hat{a}_{b o o t}-a_{b o o t}\right)$ to $\hat{a}$, where $a_{\text {boot }}=F_{\text {boot }} / \hat{M}$.

As previously discussed, $G$ is unknown in practice and estimated with $\hat{G}$. Under such circumstances a confidence interval for $a$ can be calculated by replacing $G$ with $\hat{G}$ in the above description.

Confidence intervals for $M$ can be calculated by simulating a bootstrap distribution for $\hat{M}$. For unknown $G$, this requires generating a bootstrap distribution for $\hat{G}$, which can be achieved independently of the bootstrap distribution of $\hat{a}$ (Huso et al. 2012). Given $C_{\text {boot }}, \hat{a}_{\text {boot }}$, and $\hat{G}_{\text {boot }}$, a bootstrap mortatlity estimate is calculated as,

$$
\hat{M}_{\text {boot }}=\frac{C_{\text {boot }}}{\hat{a}_{\text {boot }} \hat{G}_{b o o t}} .
$$

\section{Discussion}

The primary objective of this research was to compare five approaches to account for unsearched areas when estimating mortality at wind power facilities. A convenient example comes from defining the searched area as roads leading up to turbines, and pads beneath turbines ( $R \& P)$. We compared the methods via a simulation study that considered different R\&P configurations, spatial carcass distributions, and average carcass per turbine rates. We also proposed a semi-parametric bootstrap method that can be used to estimate uncertainty in estimates of $a$ when using the weighted distribution, cake, glm, or anisotropic glm methods.

In nearly all settings we considered, at least one, if not all, of the weighted distribution, cake, $\mathrm{glm}$, and anisotropic $\mathrm{glm}$ methods outperformed the ratio method in terms of accuracy and precision in estimating $M$. The ratio method displayed some degree of bias in every setting, which was expected because ratio estimation is always biased (Lohr 2009). In the context of turbine-induced bird and bat mortality estimation, the bias becomes increasingly prevalent as the average fatality rate of carcasses per turbine decreases.

In theory, the bias and variance of the ratio method could be reduced by clearing more turbines or increasing the probability of detection in the cleared area; however, exploratory work suggested that an unreasonable number of turbines (more than 25\%) would have to be cleared to be competitive with the other methods we considered, and increasing the probably of detection to that of the R\&P only produced modest 
increases in performance. It is interesting to note that in the exploratory research our results suggested that simply treating the data at cleared turbines as a random sample for mortality estimation produced much better results relative to using the data from the remaining $\mathrm{R} \& \mathrm{P}$ in ratio estimation.

In addition to its inferior statistical properties relative to the other methods we considered, the ratio method is also more expensive and not always possible to implement. Under the protocol used in the simulations, all methods require searching the R\&P of every turbine out to $120 \mathrm{~m}$. The weighted distribution, cake, $\mathrm{glm}$, and anisotropic glm methods also require that the facility be mapped in a GIS program before estimation. The ratio method, though, requires additional data from some plots that are cleared of vegetation. When the vegetation is dense, terrain is treacherous, or if bodies of water are within the carcass fall zone, obtaining counts for cleared turbines is impractical or even impossible. Avoiding turbines with these conditions may result in a non-representative sample of turbines. When clearing beneath turbines is possible, the cost of machine clearing as well as compensation to the land owner for lost crops add to the overall cost of the monitoring effort. In non-agricultural settings, clearing will damage the surrounding habitat, increasing the ecological impact of the wind facility. The cleared areas may also represent a substantial increase to the total area searched. At WP1 the area comprising the R\&P of all 355 turbines is equivalent to 13.1 turbines cleared to $120 \mathrm{~m}$. Therefore, under the protocol we simulated where 9 turbines were cleared and searched out to $120 \mathrm{~m}$, the ratio method requires searching $66 \%$ more area than is required for the other 4 methods. At WP2, searching the R\&P of all 23 turbines is equivalent to searching 1.8 turbines cleared to $120 \mathrm{~m}$. Therefore, under the protocol we simulated where 3 turbines were cleared and searched out to $120 \mathrm{~m}$, the ratio method requires searching $156 \%$ more area than is required for the other 4 methods.

The weighted distribution, cake, and glm methods all performed similarly in every simulation, raising the question of which method to implement in practice if it is reasonable to assume the spatial carcass distribution is isotropic. Each method possesses its own advantages.

The weighted distribution method yields a parametric fit obtained by maximizing a likelihood function, which enables comparison of parametric models, using AIC, for example. This method completely specifies the distribution of carcass distance, and allows convenient estimation of parameters such as the mean, standard deviation, relevant quantiles, and the proportion of carcasses that landed beyond the maximum distance searched. For example, when modeling the data used to generate the underlying spatial carcass distributions in the simulations along with a Weibull distribution, it is estimated that $4.7 \%$ of carcasses landed past the maximum distance searched $(90$ $\mathrm{m})$. Using a Gamma distribution, this estimate increases to $9.1 \%$. Given the ease of extending the maximum distance from the turbine searched along access roads, it is highly preferred that researchers search at distances beyond the expected maximum fall distance of a carcass, if possible, instead of relying on extrapolation beyond the range of the data. If, however, it becomes impractical to extend the plots far enough, due to resources or terrain, the parametric methods (including the weighted distribution and the glm) can be used to estimate the proportion of fatalities falling beyond 
the maximum distance searched, whereas such extrapolation is not possible with the cake or ratio methods.

The cake method estimates mortality non-parametrically allowing for relatively unbiased estimates under a variety of isotropic spatial distributions when searches are conducted to or past the maximum fall distance of a carcass. It becomes biased, however, if searches do not extend far enough, or the carcass distribution is anisotropic. The cake method proposed in this research partitions the fall zone into concentric annuli, which is reasonable under isotropic settings. It may be possible to create an anisotropic version of the cake method using the same non-parametric approach, by partitioning the fall zone into elements that are internally homogeneous with respect to the anisotropic spatial carcass distribution (see Maurer (2017)); however, determining an appropriate partition requires information about the spatial carcass distribution, which is unknown.

The $\mathrm{glm}$ model is advantageous because, unlike the weighted distribution and cake methods, which only use the univariate carcass distances, it fits a model on the observed spatial carcass distribution. The model can easily be modified to form the anisotropic $g l m$ by incorporating additional parameters that reflect dependence of carcass density on direction as well as distance. In addition to reducing bias under anisotropic spatial carcass distributions, a statistical hypothesis test can be used to determine if anisotropy is detectable by testing if terms including direction are statistically significant to the model. If there is insufficient evidence of anisotropy, then the isotropic $\mathrm{glm}$ method can be selected as the most appropriate model, and results will be similar to the weighted distribution and cake methods.

As discussed in Sect. 1, R\&P searches provide a convenient example for implementing and comparing the statistical methods discussed above. In addition, there are some logical advantages to R\&P searches relative to full-plot searches conducted by human searchers: (i) the probability of detection tends to be maximized on R\&P (Kerns et al. 2005; Good et al. 2012); (ii) searcher efficiency and carcass persistence trials only need be conducted in a single visibility class; (iii) extending the maximum distance searches increases required resources at a linear rate, if at all; and (iv) Searches can be conducted at a larger number of turbines, yielding information on spatial patterns across the entire project.

It was beyond our scope to compare these methods to a protocol that searches the entirety of a randomly selected subset of turbines when holding the total searched area constant; however, we did conduct some exploratory research. Our results suggested that under isotropic carcass distributions, estimation based on R\&P was more precise relative to searches conducted on an equivalent amount of area at cleared turbines, when the probability of detection was lower in cleared areas than on R\&P. As the probability of detection in the cleared areas approached the probability of detection on $\mathrm{R} \& \mathrm{P}$, the performance became similar. This suggests that it would behoove researchers to focus on areas with high detection probabilities before searching areas with lower detection probabilities. It should be noted that the areas with higher detection probabilities do not necessarily align perfectly with areas of higher searcher efficiency; increased visibility of carcasses to humans might apply to scavengers, as well, resulting in higher carcass removal rates and potentially lower overall detection rates in those areas. Also, the model assumes that the distribution of carcasses with respect to 
distance from turbine is the same in searched and unsearched areas. It is not likely that carcass deposition distances vary between searched and unsearched areas. However, it is possible that animal movement after hitting the ground could vary significantly by ground cover, as injured animals move from exposed areas (like roads and pads) to more protected areas before dying. The degree to which this may introduce bias in practice is unknown but may be an interesting topic for future research.

Of course, accurate and precise estimation using R\&P searches in conjunction with the methods described in this research has limitations. The weighted distribution, cake, $g l m$, and anisotropic glm methods estimate mortality by estimating the spatial carcass distribution, which requires a reasonable sample size. For example, with only 23 turbines installed at WP2, an average of 2 carcasses per turbine equated to an average of eight total carcasses observed on R\&P across all simulations. In a similar setting, the precision of the weighted distribution, cake, and $\mathrm{glm}$ methods will likely be poor, and the anisotropic glm inestimable. Therefore mortality estimation based on data solely collected on R\&P should be avoided when few carcasses are expected to be observed and precise estimates of mortality are needed. If R\&P searches are performed and few carcasses are observed, then it may be possible to use information from a nearby wind project with similar fauna, topography, wind, and turbines to estimate a spatial carcass distribution.

These methods may also fail to produce reasonable estimates if the sample of observed distances is non-representative of the underlying fall distribution. This could happen if anisotropy is present and either (i) major proportions of certain directions go unsearched, or (ii) the anisotropic carcass distribution at individual turbines interacts with the R\&P configurations. For example, (i) could occur if every turbine access road came in from the same direction. In this instance there would be no way of testing or accounting for an anisotropic carcass distribution, because of the lack of information in the other directions. Situation (ii) is possible to encounter in practice, because road engineers often take topography into consideration and winds respond to topography. This could be problematic if, for example, all access roads are parallel to prevailing wind direction, although access direction may not be constant. In this situation, the carcass locations obtained from R\&P searches would clearly not be representative. Researchers should be able to identify such situations by consulting with the engineers that built the project or using wind direction data collected at individual turbines.

Mortality estimation based entirely on R\&P searches may not be applicable in all settings, but when it is, it can provide an efficient way to gather useful estimates at a relatively low cost. The simulations preformed in this research demonstrate that it is possible to obtain accurate and precise mortality estimates from data collected entirely on R\&P searches under a wide variety of settings.

Acknowledgements We thank Cris Hein, Michael Schirmacher, Paul Rabie and Jerry Roppe for many fruitful discussions regarding the feasibility of road and pad searches, but do not wish to imply any endorsement on their part of the ideas presented here. We thank Ariel Muldoon, and two anonymous reviewers for helpful suggestions to improve the manuscript.

Funding Funding for this research was provided by the Ecosystems Mission Area Wildlife Program of the U.S. Geological Survey (USGS) and Avangrid Renewables. 
Open Access This article is licensed under a Creative Commons Attribution 4.0 International License, which permits use, sharing, adaptation, distribution and reproduction in any medium or format, as long as you give appropriate credit to the original author(s) and the source, provide a link to the Creative Commons licence, and indicate if changes were made. The images or other third party material in this article are included in the article's Creative Commons licence, unless indicated otherwise in a credit line to the material. If material is not included in the article's Creative Commons licence and your intended use is not permitted by statutory regulation or exceeds the permitted use, you will need to obtain permission directly from the copyright holder. To view a copy of this licence, visit http://creativecommons.org/licenses/by/4.0/.

\section{Appendix 1}

See Figs. 4, 5, 6, 7 and Table 4
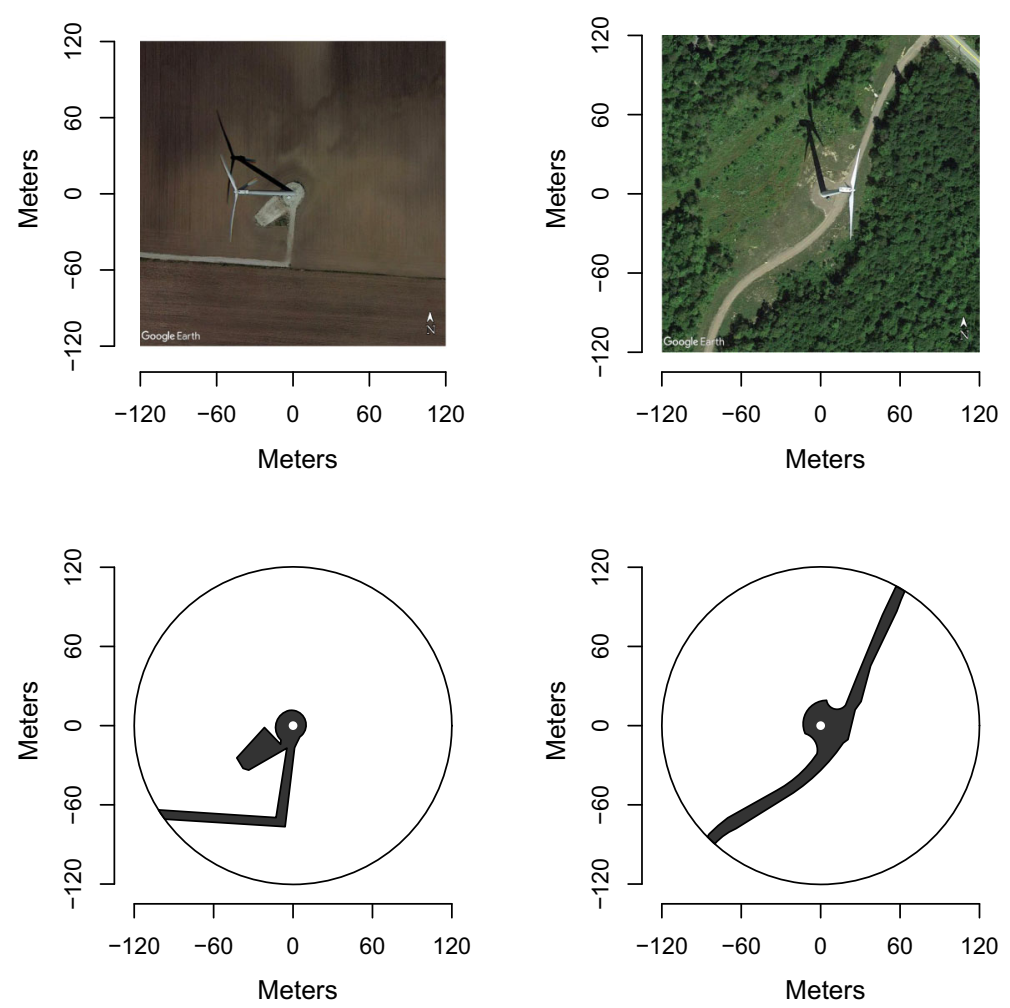

Fig. 4 Google Earth images (top; Google Earth (2012)) and R polygon plots (bottom) of a typical R\&P configuration at WP1 (left) and WP2 (right) 


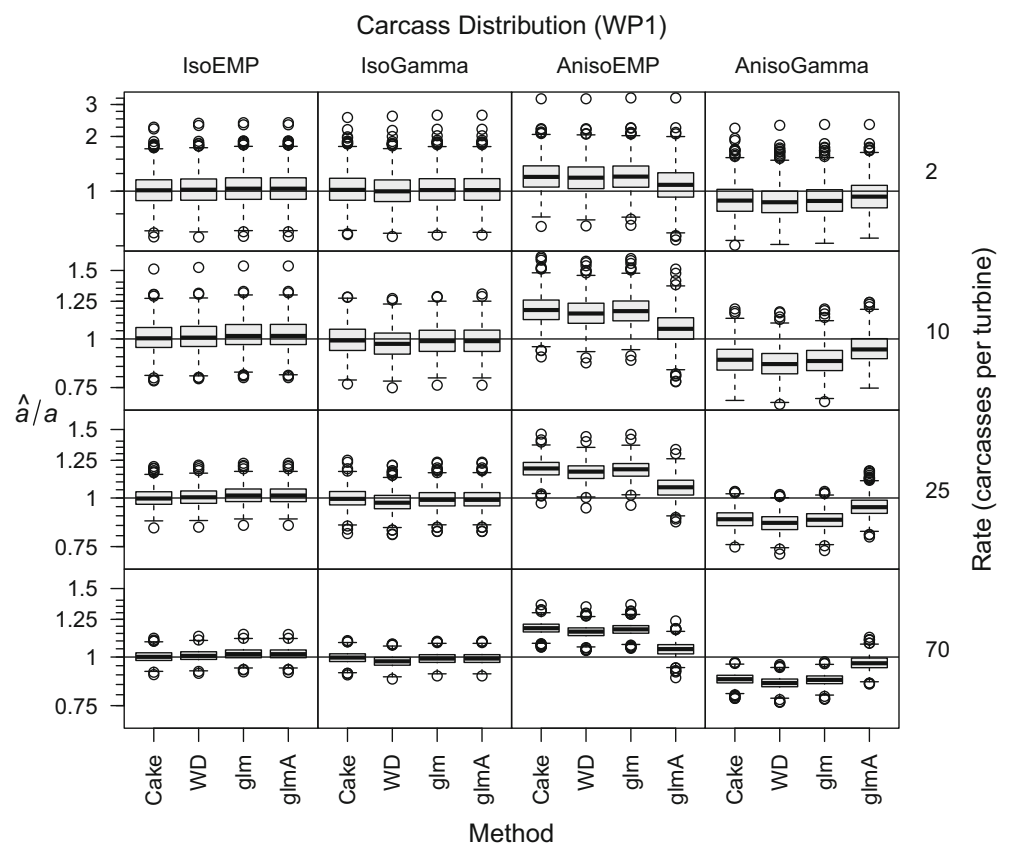

Fig. 5 Plot of $\frac{\hat{a}}{a}$ on the log scale at WP1 for each combination of average fatality per turbine rate, where $a$ is the random variable for the proportion of carcasses that landed on R\&P in a given trial. Box plots were define using default parameters in $R$, with boxes showing medians and inter-quartile ranges (IQR) and whiskers are drawn to the most extreme data points within 1.5 IQR of the 1st and 3rd quartiles 


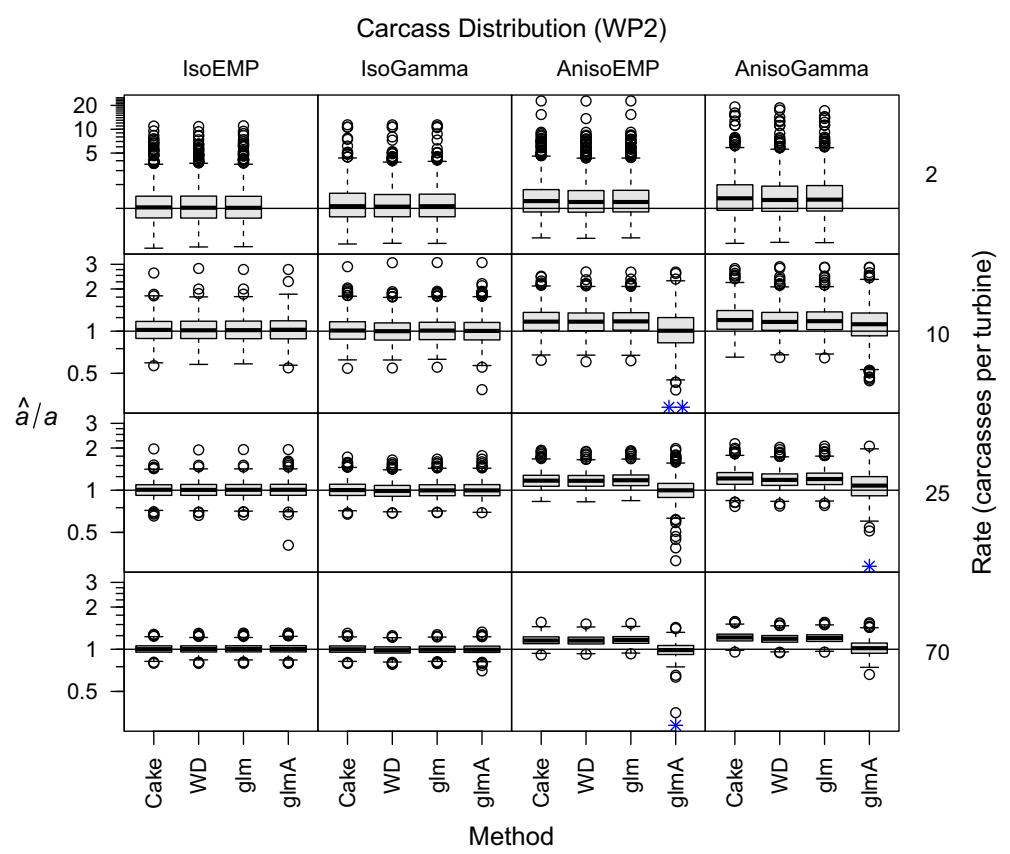

Fig. 6 Plot of $\frac{\hat{a}}{a}$ on the $\log$ scale at WP2 for each combination of average fatality per turbine rate), where $a$ is the random variable for the proportion of carcasses that landed on R\&P in a given trial. Data were insufficient for estimating the glmA model when the fatality rates were $2 /$ turbine, so box plots for those scenarios are missing
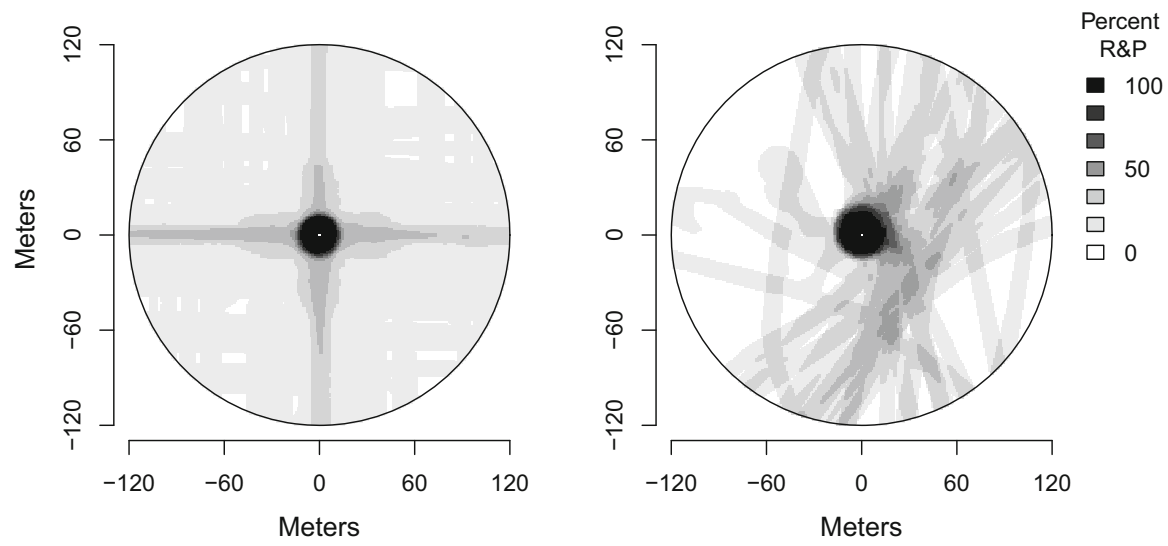

Fig. 7 Composite of R\&P configurations of all 355 turbines at WP1 (left) and 23 turbines WP2 (right). The shading for a given point is determined by the proportion of individual turbine R\&P configurations that contain that point in the R\&P. Points that are black are contained in the R\&P at every turbine, and points that are white are not contained in the R\&P of any turbine 
Table 4 Notation

\begin{tabular}{|c|c|}
\hline$a$ & Probability a carcass lands on R\&P \\
\hline$\hat{a}$ & An estimate of $a$ \\
\hline$M$ & True mortality \\
\hline$\hat{M}$ & An estimate of $M$ \\
\hline G & Probability a carcass that lands on $R \& P$ is observed \\
\hline$G_{c l r}$ & Probability a carcass that lands in an area cleared of vegetation is observed \\
\hline$\hat{G}$ & An estimate of $G$ \\
\hline$C$ & Observed number of carcasses on R\&P \\
\hline$r$ & An index indicating distance from the center of the turbine \\
\hline$r_{i}$ & Distance from the nearest turbine of the $i t h$ carcass \\
\hline$r_{\max }$ & Maximum distance from turbine searched \\
\hline$\theta$ & An index indicating direction in Azimuth from the center of a turbine \\
\hline$\theta_{i}$ & Direction in Azimuth from nearest turbine of the $i t h$ carcass \\
\hline$C_{j}$ & Observed number of carcasses in the $j t h$ annulus \\
\hline$A_{j}$ & Proportion of the $j t h$ annulus contained in R\&P (known) \\
\hline$C_{R P}$ & Number of carcasses on $R \& P$ at cleared turbines \\
\hline$C_{\text {non } R P}$ & Number of carcasses on non-R\&P at cleared turbines \\
\hline$A(r)$ & Proportion of area $r$ meters from the center of the turbine contained in R\&P \\
\hline$f(r \mid \Theta)$ & Parametric pdf of carcass distances (observed and unobserved) \\
\hline$f_{\mathrm{o}}(r \mid \Theta)$ & Parametric pdf of observed carcass distribution \\
\hline$l\left(\Theta \mid\left\{r_{1}, \ldots, r_{c}\right\}\right)$ & Log-likelihood function of $\Theta$ \\
\hline$C_{r, \theta}$ & Observed number of carcasses in cells indexed by $(r, \theta)$ \\
\hline$\lambda_{r, \theta}$ & Expected number of observed carcasses in cells index by $(r, \theta)$ \\
\hline$O_{(r, \theta)}$ & Offset for Poisson Regression $=$ Number of cells indexed by $(r, \theta)$ contained in R\&P \\
\hline$\hat{f}(r, \theta)$ & Estimated spatial carcass distribution \\
\hline$T$ & Total number of turbines \\
\hline$K$ & Order of the Fourier expansion used for the anisotropic $\mathrm{glm}$ method \\
\hline$F$ & Number of carcasses landing on $\mathrm{R} \& \mathrm{P}(F=a M)$ \\
\hline$P_{a}$ & Probability a carcass lands on $\mathrm{R} \& \mathrm{P}$ \\
\hline$F_{\text {boot }}$ & Bootstrap value for $F$ \\
\hline$C_{\text {boot }}$ & Bootstrap value for $C$ \\
\hline$a_{\text {boot }}$ & Bootstrap value of $a$ \\
\hline$\hat{a}_{\text {boot }}$ & Bootstrap estimate of $a$ \\
\hline$\hat{G}_{\text {boot }}$ & Bootstrap estimate of $G$ \\
\hline$\hat{M}_{\text {boot }}$ & Bootstrap estimate of $M$ \\
\hline
\end{tabular}




\section{References}

Akaike H (1998) Information theory and an extension of the maximum likelihood principle. In: Selected papers of Hirotugu Akaike, Springer, pp 199-213

Arnett E (2006) A preliminary evaluation on the use of dogs to recover bat fatalities at wind energy facilities. Wildl Soc Bull 34(5):1440-1445

Arnett EB, Brown W, Erickson WP, Fiedler JK, Hamilton BL, Henry TH, Jain A, Johnson GD, Kerns J, Koford RR et al (2008) Patterns of bat fatalities at wind energy facilities in North America. J Wildl Manag 72(1):61-78

Arnett E, Schirmacher M, Huso M, Hayes J (2009) Patterns of bat fatality at the Casselman Wind Project in south-central Pennsylvania. An annual report prepared for the Bats and Wind Energy Cooperative (BWEC) and the Pennsylvania Game Commission. Bat Conservation International (BCI), Austin, Texas. http://batsandwind.org/pdf/2008\%20Casselman\%20Fatality\%20Report.pdf. Accessed 16 Sept 2016

Beebe SC, Howell TJ, Bennett PC (2016) Using scent detection dogs in conservation settings: a review of scientific literature regarding their selection. Front Vet Sci 3:96

Bernardino J, Bispo R, Torres P, Rebelo R, Mascarenhas M, Costa H (2011) Enhancing carcass removal trials at three wind energy facilities in Portugal. Wildl Biol Pract 7:1-14

BHE Environmental (2011) Post-construction bird and bat mortality study, Cedar Ridge Wind Farm, Fond Du Lac County, Wisconsin. Final report. Prepared for Wisconsin Power and light. https://apps.psc.wi. gov/vs2015/erf_view/viewdoc.aspx?docid=146174. Accessed 09 Sept 2016

Bispo R, Bernardino J, Marques TA, Pestana D (2013) Modeling carcass removal time for avian mortality assessment in wind farms using survival analysis. Environ Ecol Stat 20(1):147-165

Chatfield A, Erickson W, Bay K (2009) Avian and bat fatality study, Dillon Wind-Energy Facility, Riverside County, California. Final report: March 26, 2008-March 26, (2009) Prepared for Iberdrola Renewables, Portland. Oregon. Prepared by Western EcoSystems Technology, Inc (WEST), Cheyenne, Wyoming

Dalthorp D, Huso M, Dail D (2017) Evidence of absence (v2. 0) software user guide. Tech rep, US Geological Survey. https://pubs.er.usgs.gov/publication/ds1055. Accessed 14 Aug 2018

Dalthorp DH, Simonis J, Madsen L, Huso MP, Rabie P, Mintz J, Wolpert R, Korner-Nievergelt F (2018) Genest r package. rpackage https://www.usgs.gov/software/genest-a-generalized-estimatormortality. Accessed 14 Jun 2019

Drake D, Schumacher S, Sponsler M (2012) Regional analysis of wind turbine-caused bat and bird fatality. Environmental and economic research and development program of Wisconsin's Focus on Energy, Madison, Wisconsin, USA. https://www.focusonenergy.com/sites/default/files/2018-05/1007Drake_ FinalReportx.pdf. Accessed 05 Sept 2016

Efron B, Gong G (1983) A leisurely look at the bootstrap, the jackknife, and cross-validation. Am Stat 37(1):36-48

Erickson W, Kronner K, Gritski B (2003) Nine Canyon Wind Power Project Avian and Bat Monitoring Report. Technical report submitted to Energy Northwest and Nine Canyon Technical Advisory Commitee. Western Ecosystems Technology, Inc., Cheyenne, Wyoming, USA. http://west-inc.com/ reports/nine_canyon_monitoring_final.pdf. Accessed 15 Sept 2016

Etterson MA (2013) Hidden Markov models for estimating animal mortality from anthropogenic hazards. Ecol Appl 23(8):1915-1925

Flint PL, Lance EW, Sowl KM, Donnelly TF (2010) Estimating carcass persistence and scavenging bias in a human-influenced landscape in western Alaska. J Field Ornithol 81(2):206-214

Good R, Erickson W, Merrill A, Simon S, Murray K, Bay K, Fritchman C (2011) Bat monitoring studies at the fowler ridge wind energy Facility, Benton County, Indiana: April 13-October 15, 2010. Prepared for Fowler Ridge Wind Farm. Prepared by WEST, Inc., Chyenne, Wyoming. http://batsandwind.org/ pdf/Fowler_Final_SRRP_Report_January_31_2011.pdf. Accessed 01 July 2013

Good R, Merrill A, Simon S, Murray K, Bay K (2012) Bat monitoring studies at the Fowler Ridge Wind Farm, Benton County, Indiana. An unpublished report submitted to the Fowler Ridge Wind Farm by WEST, Inc., Cheyenne, WY, USA. https://tethys.pnnl.gov/sites/default/files/publications/Good\%20et \%20al.\%202012_Fowler\%20Report.pdf. Accessed 01 July 2013

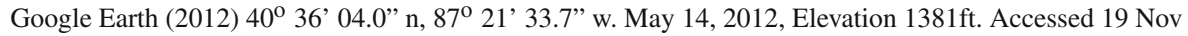
2017

Gruver J, Sonnenburg M, Bay K, Erickson W (2009) Post-construction bat and bird fatality study at the Blue Sky Green Field Wind Energy Center, Fond du Lac County, Wisconsin: July 21, 2008-October 31, 
2008 and March 15, 2009-June 4, 2009.WEST, Inc., Cheyenne, Wyoming, USA. http://batsandwind. org/pdf/Blue\%20Sky,\%20WI\%20report.pdf. Accessed 18 Sept 2016

Hallingstad EC, Rabie PA, Telander AC, Roppe JA, Nagy LR (2018) Developing an efficient protocol for monitoring eagle fatalities at wind energy facilities. PLoS ONE 13(12):e0208700

Hein CD, Prichard A, Mabee T, Schirmacher MR (2014) Efficacy of an operational minimization experiment to reduce bat fatalities at the Pinnacle Wind Farm, Mineral County, West Virginia, 2013. Final report. An annual report submitted to Edison Mission Energy and the Bats and Wind Energy Cooperative. Bat Conservation International, Austin, Texas. http://batsandwind.org/pdf/Hein\%20et\%20al.\%202014_ Operational\%20minization $\% 20$ to\%20reduce $\% 20$ bat $\% 20$ fatalities $\% 20$ in $\% 20 \mathrm{WV}$.pdf. Accessed 14 Aug 2018

Homan HJ, Linz G, Peer BD (2001) Dogs increase recovery of passerine carcasses in dense vegetation. Wildl Soc Bull 22:292-296

Howell JA, DiDonato JE (1991) Assessment of avian use and mortality related to wind turbine operations, Altamont Pass, Alameda and Contra Costa Counties, California, September 1988 through August 1989. Final report submitted to US Windpower, Inc. https://tethys.pnnl.gov/sites/default/files/publications/ Howell-1991.pdf. Accessed 05 Sept 2016

Hull C, Muir S (2010) Search areas for monitoring bird and bat carcasses at wind farms using a Monte-Carlo model. Australas J Environ Manag 17(2):77-87

Huso MM (2011) An estimator of wildlife fatality from observed carcasses. Environmetrics 22(3):318-329

Huso MMP, Dalthorp D (2014) Accounting for unsearched areas in estimating wind turbine-caused fatality. J Wildl Manag 78(2):347-358

Huso MMP, Som N, Ladd L (2012) Fatality estimator user's guide. Tech rep, US Geological Survey, https:// pubs.usgs.gov/ds/729/pdf/ds729.pdf. Accessed 14 Aug 2018

Huso MMP, Dalthorp D, Korner-Nievergelt F (2017) Statistical principles of post-construction fatality monitoring design. Wildlife and wind farms, conflicts and solutions 2:84-103. In Perrow M (ed) Onshore: Monitoring and Mitigation. Pelagic Publishing, Exeter

Jain AA (2005) Bird and bat behavior and mortality at a northern Iowa windfarm. Iowa State University, Ames, Iowa, USA. Master's thesis. http://batsandwind.org/pdf/Jain_2005.pdf. Accessed 29 June 2013

Kerns J, Kerlinger P (2004) A study of bird and bat collision fatalities at the Mountaineer Wind Energy Center, Tucker County, West Virginia: Annual report for 2003. Prepared for FPL Energy and Mountaineer Wind Energy Center Technical Review Committee. Curry and Kerlinger L. L. C., McLean, Virginia, USA. http://www.fwspubs.org/doi/suppl/10.3996/032012-JFWM-024/suppl_file/10.3996_ 032012-jfwm-024.s1.pdf?code=ufws-site

Kerns J, Erickson WP, Arnett EB (2005) Bat and bird fatality at wind energy facilities in Pennsylvania and West Virginia. Relationships between bats and wind turbines in Pennsylvania and West Virginia: an assessment of fatality search protocols, patterns of fatality, and behavioral interactions with wind turbines. The Bats and Wind Energy Cooperative, Bat Conservation International, Austin, Tex, USA. pp 24-95. http://batsandwind.org/pdf/postconpatbatfatal.pdf

Korner-Nievergelt F, Brinkmann R, Niermann I, Behr O (2013) Estimating bat and bird mortality occurring at wind energy turbines from covariates and carcass searches using mixture models. PLoS ONE 8(7):e67997

Korner-Nievergelt F, Behr O, Brinkmann R, Etterson MA, Huso MM, Dalthorp D, Korner-Nievergelt P, Roth T, Niermann I (2015) Mortality estimation from carcass searches using the R-package carcass-a tutorial. Wildl Biol 21(1):30-43

Kunz TH, Arnett EB, Erickson WP, Hoar AR, Johnson GD, Larkin RP, Strickland MD, Thresher RW, Tuttle MD (2007) Ecological impacts of wind energy development on bats: questions, research needs, and hypotheses. Front Ecol Environ 5(6):315-324

Lohr S (2009) Sampling: design and analysis. Nelson Education, Toronto

Mathews F, Swindells M, Goodhead R, August TA, Hardman P, Linton DM, Hosken DJ (2013) Effectiveness of search dogs compared with human observers in locating bat carcasses at wind-turbine sites: A blinded randomized trial. Wildlife Society Bulletin 37(1):34-40

Maurer J (2017) Turbine induced bird and bat fatalities at wind projects: statistical methods for mortality estimation using road and pad CarcassSurveys. PhD thesis, Oregon State University, Corvallis, OR, USA

Orloff S, Flannery A (1992) Wind turbine effects on avian activity, habitat use, and mortality in altamont pass and solano country wind resource areas: 1989-1991. Final report to Alameda, Costra Costa and 
Solano Counties and the Califorina Energy Commission by Bio systems Analysis Inc., Tiburon, CA. https://tethys.pnnl.gov/sites/default/files/publications/Orloff-1992.pdf. Accessed 30 June 2018

Patil GP, Rao CR (1978) Weighted distributions and size-biased sampling with applications to wildlife populations and human families. Biometrics 34:179-189

Pewsey A, Neuhäuser M, Ruxton GD (2013) Circular statistics in R. Oxford University Press, Oxford

R Core Team (2015) R: a language and environment for statistical computing. R Foundation for Statistical Computing, Vienna, Austria. http://www.R-project.org/

Reyes GA, Rodriguez MJ, Lindke KT, Ayres KL, Halterman MD, Boroski BB, Johnston DS (2016) Searcher efficiency and survey coverage affect precision of fatality estimates. J Wildl Manag 80(8):1488-1496

Shoenfeld P (2004) Suggestions regarding avian mortality extrapolation. Prepared for the Mountaineer Wind Energy Center Technical Review Committee. http://www.wvhighlands.org/Birds/ SuggestionsRegardingAvianMortalityExtrapolation.pdf. Accessed 13 Sept 2016

Strickland M, Arnett E, Erickson W, Johnson D, Johnson G, Morrison M, Shaffer J, Warren-Hicks W (2011) Comprehensive guide to studying wind energy/wildlife interactions. Prepared for the National Wind Coordinating Collaborative, Washington, DC, USA. https://tethys.pnnl.gov/sites/default/files/ publications/Comprehensive-Guide-to-Studying-Wind-Energy-Wildlife-Interactions.pdf. Accessed 14 Aug 2018

Studyvin J, Rabie P, Riser-Espinoza D, McDonald TL (2019) windac: area corrections methods. r package https://CRAN.R-project.org/package=windAC. Accessed 14 Nov 2019

Thompson SK (2012) Sampling. Wiley, Hoboken

Turner KL, Abernethy EF, Mike Conner L, Rhodes OE, Beasley JC (2017) Abiotic and biotic factors modulate carrion fate and vertebrate scavenging communities. Ecology 98(9):2413-2424

US Fish and Wildlife Service (2012) US Fish and Wildlife Service land-based wind energy guidelines. https://www.fws.gov/ecological-services/es-library/pdfs/WEG_final.pdf. Accessed 07 Oct 2014

Warton DI, Shepherd LC et al (2010) Poisson point process models solve the (pseudo-absence problem) for presence-only data in ecology. Ann Appl Stat 4(3):1383-1402

Wolpert R (2013) Appendix b: a partially periodic equation for estimating avian mortality rates. Improving methods for estimating fatality of birds and bats at wind energy facilities, California Wind Energy Association, Berkeley, California, USA

Wolpert RL (2015) ACME: a partially periodic estimator of avian \& chiropteran mortality at wind turbines. arXiv preprint arXiv:1507.00749

Joseph Maurer is currently an industrial statistician for W. L. Gore and Associates. The research presented in this article was largely conducted as part of the requirements for his doctoral degree. Joseph Maurer graduated with his Ph.D. in Statistics from Oregon State University in August 2017.

Manuela Huso is a Research Statistician with the USGS in Corvallis, OR and affiliate faculty in the Department of Statistics at Oregon State University. She is coauthor of several mortality estimators: carcass, DS729, GenEst and EoA and has conducted studies testing curtailment and deterrents for reducing bat mortality.

Dan Dalthorp received the A.B degree in Mathematics from Brown University in 1986, the M.S. degree in Mathematics from the University of Oregon in 1989, the M.S. degree in Environmental Systems from Humboldt State University in 1993, and the Ph.D degree from Cornell University in 1999. He is currently working as a statistician at USGS in Corvallis, Oregon. His research interests include population ecology, spatial statistics, and estimation of bird and bat mortality at commercial renewable energy facilities.

Lisa Madsen received the B.S. degree in Mathematics and Computer Science from University of Oregon, Eugene, Oregon, USA, in 1986, the M.S. degree in Mathematics from University of Oregon, Eugene, Oregon, USA, in 1990, the M.S. degree in Statistics from Cornell University, Ithaca, New York, USA in 2000, and the Ph.D. degree in Statistics from Cornell University, Ithaca, New York, USA in 2004. She is an associate professor at Oregon State University, Corvallis, Oregon, USA. Her research interests include dependent non-Gaussian data, abundance and occupancy models, spatial statistics, and ecological and environmental statistics. 
Claudio Fuentes received his B.S. degree in Mathematics in 2001 from the Pontifical Catholic University of Chile, Santiago, Chile, and a M.S. in Statistics from the same institution in 2003. Later on he continued his education in the United States where earned his M.S. degree in Statistics in 2008 and the Ph.D. in Statistics in 2011 from the University of Florida, Gainesville, Florida, USA. After completing his Ph.D. he joined the Department of Statistics at Oregon State University, Corvallis, Oregon, USA, where he continues his academic activities as an Associate Professor. His research interests include clustering and classification problems, post-selection inference and Bayesian methodology. 